\title{
Large deviations for the stochastic shell model of turbulence
}

\author{
U. Manna, S. S. Sritharan and P. Sundar
}

\begin{abstract}
In this work, we first prove the existence and uniqueness of a strong solution to stochastic GOY model of turbulence with a small multiplicative noise. Then using the weak convergence approach, Laplace principle for solutions of the stochastic GOY model is established in certain Polish space. Thus a Wentzell-Freidlin type large deviation principle is established utilizing certain results by Varadhan and Bryc.
\end{abstract}

Mathematics Subject Classification (2000). Primary 60F10; Secondary 60H15, 76D03, 76D06.

Keywords. GOY model, Large deviations, Local monotonicity.

\section{Introduction}

The large deviations theory is among the most classical areas in probability theory with many deep developments and applications $[10,11,13,17,35,37]$. Although it appears to be no literature on the large deviation results for stochastic shell model of turbulence, a few authors have proved the Wentzell-Freidlin type large deviations for the two-dimensional stochastic Navier-Stokes equations with additive noise (e.g. [6]) and also for multiplicative noise (e.g. [34]). For Donsker-Varadhan type large deviation study related to Navier-Stokes equations we refer the readers to Quastel and Yau [32]. For the treatment related to stochastic two-dimensional vorticity equations see the work of Amirdjanova and Xiong [1]. Several authors have established the Wentzell-Freidlin type large deviation estimates for a class of infinite dimensional stochastic differential equations (see for e.g., $[4,7,9,20,33]$ ). In these works the proofs of large deviation principle (LDP) (see Definition 2.2) usually rely on first approximating the original problem by time-discretization so that LDP can be shown for the resulting simpler problems via contraction principle, and then showing that LDP holds in the limit. The discretization method to establish LDP was introduced by Wentzell and Freidlin[17]. 
Dupuis and Ellis [13] have combined weak convergence methods to the stochastic control approach developed earlier by Fleming [16] to the large deviations theory. Our work is based on the theory introduced by Budhiraja and Dupuis [4], where they used the stochastic control and weak convergence approach to obtain the LDP for the family $\left\{\mathcal{G}^{\varepsilon}(W(\cdot))\right\}_{\varepsilon>0}$, where $\mathcal{G}^{\varepsilon}$ is an appropriate family of measurable maps from the Wiener space to some Polish space. Their work relied on the fact that the LDP is equivalent to Laplace principle (see Definition 2.6) if the underlying space is Polish, which is in turn a consequence of Varadhan's Lemma (see Lemma 2.7) and Bryc's converse to Varadhan's Lemma (see Lemma 2.8). We refer the reader to Theorems 1.2.1 and 1.2.3 in Dupuis and Ellis [13].

This work deals with an infinite dimensional shell model, a mathematical turbulence model that received increasing attention in recent years. Apparently there are only two previous rigorous works on infinite dimensional shell model, namely [8] and [2], one in the deterministic case and the other in the stochastic case with additive noise, respectively. In both of these works a variational semigroup formulation has been introduced. Our present work deals with a more general stochastic model, with multiplicative noise: the proofs of existence and uniqueness of strong solutions are considerably more difficult in this case. The main result of this paper is to prove a large deviation principle for the solution of the shell model.

The construction of the paper is as follows. In the next section, we give some definitions and basic properties from the large deviation theory. In later part of this section, we describe briefly the work of Budhiraja and Dupuis [4] to set up the ground for our main work. In Sect. 3, we formulate the abstract stochastic GOY model when the noise coefficient is small. We then prove certain a priori energy estimates with exponential weight. These estimates together with the local monotonicity property of the sum of the linear and non linear operators play a fundamental role to prove the existence and uniqueness of the strong solution. In the last section, we establish the LDP for the stochastic GOY model perturbed by a small multiplicative noise.

\section{Large deviation principle}

In this section, we will give an abstract formulation and basic properties for a class of large deviation problems. Let us denote by $X$ a complete separable metric space and $\left\{P_{\varepsilon}: \varepsilon>0\right\}$ a family of probability measures on the Borel subsets of $X$.

Definition 2.1. A function $I: X \rightarrow[0, \infty]$ is called a rate function if $I$ is lower semicontinuous. A rate function $I$ is called a good rate function if for arbitrary $M \in[0, \infty)$, the level set $K_{M}=\{x: I(x) \leq M\}$ is compact in $X$

Definition 2.2. (Large Deviation Principle) We say that a family of probability measures $\left\{P_{\varepsilon}: \varepsilon>0\right\}$ satisfies the large deviation principle (LDP) with a good rate function $I$ satisfying, 
(i) for each closed set $F \subset X$

$$
\limsup _{\varepsilon \rightarrow 0} \varepsilon \log P_{\varepsilon}(F) \leq-\inf _{x \in F} I(x),
$$

(ii) for each open set $G \subset X$

$$
\liminf _{\varepsilon \rightarrow 0} \varepsilon \log P_{\varepsilon}(G) \geq-\inf _{x \in G} I(x) .
$$

Remark 2.3. For any given $\left\{P_{\varepsilon}: \varepsilon>0\right\}$ there is at most one rate function governing the large deviations of $\left\{P_{\varepsilon}: \varepsilon>0\right\}$.

Example 2.4. As a simplest example we recall the one dimensional version of Cramér's theorem [37]. Let $X_{n}=\left(Y_{1}+Y_{2}+\cdots+Y_{n}\right) / n$ where $\left\{Y_{j}\right\}$ are independent random variables with a common distribution $\alpha$. Assume that the moment generating function

$$
M(\theta)=E[\exp (\theta Y)]=\int e^{\theta y} \mathrm{~d} \alpha(y)
$$

is finite for all $\theta$. Then $\left\{X_{n}\right\}$ satisfies the LDP with rate function (see [11])

$$
I(x)=\sup _{\theta}[\theta x-\log M(\theta)] .
$$

Example 2.5. We choose Schilder's theorem as second example which has many important applications in large deviation theory, such as, in the derivation of the Strassen's renowned Law of Iterated Logarithm, in the Wentzell and Freidlin's estimate on the large deviations of randomly perturbed dynamical systems, to name a few.

Let $d \in \mathbb{Z}^{+}$and

$$
A_{0}=\left\{\phi \in \mathcal{C}\left([0, \infty) ; \mathbb{R}^{d}\right): \phi(0)=0 \text { and } \lim _{t \rightarrow \infty} \frac{|\phi(t)|}{t}=0\right\} .
$$

For $\phi \in A_{0}$ define

$$
\|\phi\|_{A_{0}}=\sup _{t \geq 0} \frac{|\phi(t)|}{1+t} .
$$

Then notice that $A_{0}$ is a separable real Banach space [13]. Next, we define $\mathrm{H}^{1}=\mathrm{H}^{1}\left([0, \infty) ; \mathbb{R}^{d}\right)$ to be the space of $\phi \in A_{0}$ with the property that $\phi(t)=$ $\int_{0}^{t} \dot{\phi}(s) \mathrm{d} s, t \geq 0$, for some $\dot{\phi} \in \mathrm{L}^{2}\left([0, \infty) ; \mathbb{R}^{d}\right)$ and set $\|\phi\|_{\mathrm{H}^{1}}=\|\dot{\phi}\|_{\mathrm{L}^{2}\left([0, \infty) ; \mathbb{R}^{d}\right)}$, for $\phi \in \mathrm{H}^{1}$.

Now for given $T>0$, we define $I_{T}: A_{0} \rightarrow[0, \infty]$ by

$$
I_{T}(\psi)= \begin{cases}\frac{1}{2} \int_{0}^{T}|\dot{\psi}(t)|^{2} \mathrm{~d} t & \text { if } \psi \in \mathrm{H}^{1}, \\ \infty & \text { otherwise. }\end{cases}
$$

Let $\{W(t)\}$ be a standard Wiener process in $\mathbb{R}^{d}$. Let the process

$$
X_{n}(t)=\frac{1}{\sqrt{n}} W(t)
$$


takes values in a Polish space $E$. Then $\left\{X_{n}\right\}$ satisfies the LDP on $E$ with the rate function $I_{T}$ (see [13]).

Definition 2.6. (Laplace Principle) For $h \in C_{b}([0,1])$,

$$
\lim _{n \rightarrow \infty} \frac{1}{n} \log \int_{0}^{1} e^{-n h(x)} \mathrm{d} x=-\min _{x \in[0,1]} h(x) .
$$

Lemma 2.7. (Varadhan's Lemma [37]) Let $E$ be a Polish space and $\left\{X^{\varepsilon}: \varepsilon>0\right\}$ be a family of E-valued random elements satisfying $L D P$ with rate function $I$. Then $\left\{X^{\varepsilon}: \varepsilon>0\right\}$ satisfies the Laplace principle on $E$ with the same rate function $I$ if for all $h \in C_{b}(E)$,

$$
\lim _{\varepsilon \rightarrow 0} \varepsilon \log E\left\{\exp \left[-\frac{1}{\varepsilon} h\left(X^{\varepsilon}\right)\right]\right\}=-\inf _{x \in E}\{h(x)+I(x)\} .
$$

Lemma 2.8. (Bryc's Lemma [13]) The Laplace principle implies the LDP with the same rate function. More precisely, if $\left\{X^{\varepsilon}: \varepsilon>0\right\}$ satisfies the Laplace principle on the Polish space $E$ with the rate function $I$ and if the limit

$$
\lim _{\varepsilon \rightarrow 0} \varepsilon \log E\left\{\exp \left[-\frac{1}{\varepsilon} h\left(X^{\varepsilon}\right)\right]\right\}=-\inf _{x \in E}\{h(x)+I(x)\}
$$

is valid for all $h \in C_{b}(E)$, then $\left\{X^{\varepsilon}: \varepsilon>0\right\}$ satisfies the $L D P$ on $E$ with rate function $I$.

Note that, Varadhan's Lemma together with Bryc's converse of Varadhan's Lemma state that for Polish space valued random elements, the Laplace principle and the large deviation principle are equivalent.

Let $(\Omega, \mathcal{F}, P)$ be a probability space equipped with an increasing family $\left\{\mathcal{F}_{t}\right\}_{0 \leq t \leq T}$ of sub-sigma-fields of $\mathcal{F}$ satisfying the usual conditions of right continuity and $P$-completeness. Let $H$ be a real separable Hilbert space and $Q$ be a strictly positive, symmetric, trace class operator on $H$.

Definition 2.9. A stochastic process $\{W(t)\}_{0 \leq t \leq T}$ is said to be an $H$-valued $\mathcal{F}_{t^{-}}$ adapted Wiener process with covariance operator $Q$ if

(1) For each non-zero $h \in H,\left|Q^{1 / 2} h\right|^{-1}(W(t), h)$ is a standard one-dimensional

Wiener process,

(2) For any $h \in H,(W(t), h)$ is a martingale adapted to $\mathcal{F}_{t}$.

If $W$ is a an $H$-valued Wiener process with covariance operator $Q$ with $\operatorname{Tr} Q<\infty$, then $W$ is a Gaussian process on $H$ and

$$
E(W(t))=0, \quad \operatorname{Cov}(W(t))=t Q, \quad t \geq 0 .
$$

Let $H_{0}=Q^{1 / 2} H$. Then $H_{0}$ is a Hilbert space equipped with the inner product $(\cdot, \cdot)_{0}$,

$$
(u, v)_{0}=\left(Q^{-1 / 2} u, Q^{-1 / 2} v\right), \quad \forall u, v \in H_{0},
$$

where $Q^{-1 / 2}$ is the pseudo-inverse of $Q^{1 / 2}$. Since $Q$ is a trace class operator, the imbedding of $H_{0}$ in $H$ is Hilbert-Schmidt. 
Let $L_{Q}$ denote the space of linear operators $S$ such that $S Q^{1 / 2}$ is a HilbertSchmidt operator from $H$ to $H$. Define the norm on the space $\mathrm{L}_{Q}$ by $|S|_{\mathrm{L}_{Q}}^{2}=$ $\operatorname{Tr}\left(S Q S^{*}\right)$.

Let

$\mathcal{A}=\left\{H_{0}\right.$-valued $\left\{\mathcal{F}_{t}\right\}$-predictableprocesses $v$ such that $\int_{0}^{T}|v(s)|_{0}^{2} \mathrm{~d} s<\infty$ a.s $\}$.

Define the set $S_{N}$ of bounded deterministic controls as,

$$
S_{N}=\left\{v \in \mathrm{L}^{2}\left([0, T] ; H_{0}\right): \int_{0}^{T}|v(s)|_{0}^{2} \mathrm{~d} s \leq N\right\} .
$$

The set $S_{N}$ endowed with the weak topology on $\mathrm{L}^{2}\left([0, T] ; H_{0}\right)$ is a Polish space $[12]$.

Define $\mathcal{A}_{N}$ as the set of bounded stochastic controls by

$$
\mathcal{A}_{N}=\left\{v \in \mathcal{A}: v(\omega) \in S_{N}, \text { P-a.s. }\right\} .
$$

Let $E$ denote a Polish space, and for $\varepsilon>0$ let $\mathcal{G}^{\varepsilon}: C([0, T] ; H) \rightarrow E$ be a measurable map. Define

$$
X^{\varepsilon}=\mathcal{G}^{\varepsilon}(W(\cdot)) .
$$

We are interested in the large deviation principle for $X^{\varepsilon}$ as $\varepsilon \rightarrow 0$.

Assumption 2.10. There exists a measurable map $\mathcal{G}^{0}: C([0, T]: H) \rightarrow E$ such that the following hold:

1. Let $\left\{v^{\varepsilon}: \varepsilon>0\right\} \subset \mathcal{A}_{M}$ for some $M<\infty$. Let $v^{\varepsilon}$ converge in distribution as $S_{M}$-valued random elements to $v$. Then $\mathcal{G}^{\varepsilon}\left(W(\cdot)+\frac{1}{\sqrt{\varepsilon}} \int_{0}^{\cdot} v^{\varepsilon}(s) \mathrm{d} s\right)$ converges in distribution to $\mathcal{G}^{0}\left(\int_{0} v(s) \mathrm{d} s\right)$.

2. For every $M<\infty$, the set

$$
K_{M}=\left\{\mathcal{G}^{0}\left(\int_{0}^{\cdot} v(s) \mathrm{d} s\right): v \in S_{M}\right\}
$$

is a compact subset of $E$.

For each $f \in E$, define

$$
I(f)=\inf _{\left\{v \in \mathrm{L}^{2}\left([0, T] ; H_{0}\right): f=\mathcal{G}^{0}\left(\int_{0} v(s) \mathrm{d} s\right)\right\}}\left\{\frac{1}{2} \int_{0}^{T}|v(s)|_{0}^{2} \mathrm{~d} s\right\}
$$

where infimum over an empty set is taken as $\infty$.

We now state an important result by Budhiraja and Dupuis [4].

Theorem 2.11. Let $X^{\varepsilon}=\mathcal{G}^{\varepsilon}(W(\cdot))$. If $\left\{\mathcal{G}^{\varepsilon}\right\}$ satisfies the Assumption (2.10), then the family $\left\{X^{\varepsilon}: \varepsilon>0\right\}$ satisfies the Laplace principle in $E$ with rate function $I$ given by $(2.3)$.

Remark 2.12. 1. Notice that since the underlying space $E$ is Polish, the family $\left\{X^{\varepsilon}: \varepsilon>0\right\}$ satisfies the LDP in $E$ with the same rate function $I$. 
2. Assumption 1. is a statement on the weak convergence of a certain family of random variables and is at the core of weak convergence approach to the study of large deviations. Assumption 2. essentially says that the level sets of the rate function are compact.

\section{The stochastic GOY model of turbulence}

The GOY model (Gledger-Ohkitani-Yamada) [29] is a particular case of so called "Shell model" (see [18]). This model is the Navier-Stokes equation written in the Fourier space where the interaction between different modes is preserved between nearest modes. To be precise, the GOY model describes a one-dimensional cascade of energies among an infinite sequence of complex velocities, $\left\{u_{n}(t)\right\}$, on a one-dimensional sequence of wave numbers

$$
k_{n}=k_{0} 2^{n}, \quad k_{0}>0, \quad n=1,2, \ldots
$$

where the discrete index $n$ is referred to as the "shell index". The equations of motion of the stochastic GOY model of turbulence have the form

$$
\begin{aligned}
& \frac{\mathrm{d} u_{n}}{\mathrm{~d} t}+\nu k_{n}^{2} u_{n}+i\left(a k_{n} u_{n+1}^{\star} u_{n+2}^{\star}+b k_{n-1} u_{n-1}^{\star} u_{n+1}^{\star}\right. \\
& \left.\quad+c k_{n-2} u_{n-1}^{\star} u_{n-2}^{\star}\right)=f_{n}+\sigma_{n}\left(t, u_{n}\right) \frac{\mathrm{d} w_{n}(t)}{\mathrm{d} t}, \quad \text { for } n=1,2, \ldots,
\end{aligned}
$$

along with the boundary conditions

$$
u_{-1}=u_{0}=0 .
$$

Here $u_{n}^{\star}$ denotes the complex conjugate of $u_{n}, \nu>0$ is the kinematic viscosity and $f_{n}$ is the Fourier component of the forcing. $a, b$ and $c$ are real parameters such that energy conservation condition $a+b+c=0$ holds (see [19,29]). For the standard model $a=-1, b=1 / 2$ and $c=1 / 2$. For each $n, w_{n}$ is one-dimensional Brownian motion and the noise coefficient $\sigma_{n}$ is assumed to satisfy the following properties,

a.1. For all $t \in[0, T]$, there exists a positive constant $K_{1}$ independent of $t$ and $n$ such that,

$$
\left|\sigma_{n}\left(t, u_{n}\right)\right|^{2} \leq K_{1} k_{n}^{2}\left|u_{n}\right|^{2},
$$

a.2. For all $t \in[0, T]$, there exists a positive constant $K_{2}$ independent of $t$ and $n$ such that,

$$
\left|\sigma_{n}\left(t, u_{n}\right)-\sigma_{n}\left(t, v_{n}\right)\right|^{2} \leq K_{2} k_{n}^{2}\left|u_{n}-v_{n}\right|^{2}
$$

\subsection{Functional setting}

Let $H$ be a real Hilbert space such that

$$
H:=\left\{u=\left(u_{1}, u_{2}, \ldots\right) \in \mathbb{C}^{\infty}: \sum_{n=1}^{\infty}\left|u_{n}\right|^{2}<\infty\right\} .
$$


For every $u, v \in H$, the scalar product $(\cdot, \cdot)$ and norm $|\cdot|$ are defined on $H$ as

$$
(u, v)_{H}=\operatorname{Re} \sum_{n=1}^{\infty} u_{n} v_{n}^{\star}, \quad|u|=\left(\sum_{n=1}^{\infty}\left|u_{n}\right|^{2}\right)^{1 / 2} .
$$

Let us now define the space

$$
V:=\left\{u \in H: \sum_{n=1}^{\infty} k_{n}^{2}\left|u_{n}\right|^{2}<\infty\right\},
$$

which is a Hilbert space equipped with the norm

$$
\|u\|=\left(\sum_{n=1}^{\infty} k_{n}^{2}\left|u_{n}\right|^{2}\right)^{1 / 2} .
$$

The linear operator $A: D(A) \rightarrow H$ is a positive definite, self adjoint linear operator defined by

$$
A u=\left((A u)_{1},(A u)_{2}, \ldots\right), \text { where }(A u)_{n}=k_{n}^{2} u_{n}, \quad \forall u \in D(A) .
$$

The domain of $A, D(A) \subset H$, is a Hilbert space equipped with the norm

$$
\|u\|_{D(A)}=|A u|=\left(\sum_{n=1}^{\infty} k_{n}^{4}\left|u_{n}\right|^{2}\right)^{1 / 2}, \quad \forall u \in D(A) .
$$

Since the operator $A$ is positive definite, we can define the power $A^{1 / 2}$,

$$
A^{1 / 2} u=\left(k_{1} u_{1}, k_{2} u_{2}, \ldots\right), \quad \forall u=\left(u_{1}, u_{2}, \ldots\right) .
$$

Furthermore, we define the space

$$
D\left(A^{1 / 2}\right)=\left\{u=\left(u_{1}, u_{2}, \ldots\right): \sum_{n=1}^{\infty} k_{n}^{2}\left|u_{n}\right|^{2}<\infty\right\}
$$

which is a Hilbert space equipped with the scalar product

$$
(u, v)_{D\left(A^{1 / 2}\right)}=\left(A^{1 / 2} u, A^{1 / 2} v\right), \quad \forall u, v \in D\left(A^{1 / 2}\right),
$$

and the norm

$$
\|u\|_{D\left(A^{1 / 2}\right)}=\left(\sum_{n=1}^{\infty} k_{n}^{2}\left|u_{n}\right|^{2}\right)^{1 / 2} .
$$

Note that $V=D\left(A^{1 / 2}\right)$. We consider $V^{\prime}=D\left(A^{-1 / 2}\right)$ as the dual space of $V$. Then the following inclusion holds

$$
V \subset H=H^{\prime} \subset V^{\prime} .
$$


We will now introduce the sequence spaces analogue to Sobolev functional spaces. For $1 \leq p<\infty$ and $s \in \mathbb{R}$

$$
\mathrm{W}^{s, p}:=\left\{u=\left(u_{1}, u_{2}, \ldots\right):\left\|A^{s / 2} u\right\|_{p}=\left(\sum_{n=1}^{\infty}\left(k_{n}^{s}\left|u_{n}\right|\right)^{p}\right)^{1 / p}<\infty\right\},
$$

and for $p=\infty$

$$
\mathrm{W}^{s, \infty}:=\left\{u=\left(u_{1}, u_{2}, \ldots\right):\left\|A^{s / 2} u\right\|_{\infty}=\sup _{1 \leq n<\infty}\left(k_{n}^{s}\left|u_{n}\right|\right)<\infty\right\},
$$

where for $u \in \mathrm{W}^{s, p}$ the norm is defined as

$$
\|u\|_{\mathrm{W}^{s, p}}=\left\|A^{s / 2} u\right\|_{p} .
$$

Here $\|\cdot\|$ denotes the usual norm in the $l^{p}$ sequence space. It is clear from the above definitions that $W^{1,2}=V=D\left(A^{1 / 2}\right)$.

Remark 3.1. For the shell model we can reasonably assume that the complex velocities $u_{n}$ are such that $\left|u_{n}\right|<1$ for almost all $n$. Then

$$
\|u\|_{l^{4}}^{4}=\sum_{n=1}^{\infty}\left|u_{n}\right|^{4} \leq\left(\sum_{n=1}^{\infty}\left|u_{n}\right|^{2}\right)^{2}=|u|^{4},
$$

which leads to $H \subset l^{4}$.

We now state a Lemma which is useful in this work. We omit the proof since it is quite simple.

Lemma 3.2. For any smooth function $u \in H$, the following holds:

$$
\|u\|_{l^{4}}^{4} \leq C|u|^{2}\|u\|^{2} \text {. }
$$

\subsection{Properties of the linear and nonlinear operators}

We define the bilinear operator $B(\cdot, \cdot): V \times H \rightarrow H$ as

$$
B(u, v)=\left(B_{1}(u, v), B_{2}(u, v), \ldots\right),
$$

where

$$
B_{n}(u, v)=i k_{n}\left(\frac{1}{4} u_{n+1}^{\star} v_{n-1}^{\star}-\frac{1}{2}\left(u_{n+1}^{\star} v_{n+2}^{\star}+u_{n+2}^{\star} v_{n+1}^{\star}\right)+\frac{1}{8} u_{n-1}^{\star} v_{n-2}^{\star}\right) .
$$

In other words, if $\left\{e_{n}\right\}_{n=1}^{\infty}$ be a orthonormal basis of $H$, i.e. all the entries of $e_{n}$ are zero except at the place $n$ it is equal to 1 , then

$$
B(u, v)=i \sum_{n=1}^{\infty} k_{n}\left(\frac{1}{4} u_{n+1}^{\star} v_{n-1}^{\star}-\frac{1}{2}\left(u_{n+1}^{\star} v_{n+2}^{\star}+u_{n+2}^{\star} v_{n+1}^{\star}\right)+\frac{1}{8} u_{n-1}^{\star} v_{n-2}^{\star}\right) e_{n} .
$$

The following lemma says that $B(u, v)$ makes sense as an element of $H$, whenever $u \in V$ and $v \in H$ or $u \in H$ and $v \in V$. It also says that $B(u, v)$ makes sense as an element of $V^{\prime}$. Here we state the following lemma which has been 
proved in Constantin et al. [8] for the Sabra shell model, but one can also prove the similar estimates for the GOY model (see [2]).

Lemma 3.3. (i) There exist constants $C_{1}>0, C_{2}>0$,

$$
|B(u, v)| \leq C_{1}\|u\||v|, \quad \forall u \in V, v \in H,
$$

and

$$
|B(u, v)| \leq C_{2}|u|\|v\|, \quad \forall u \in H, v \in V .
$$

(ii) $B: H \times H \rightarrow V^{\prime}$ is a bounded bilinear operator and for a constant $C_{3}>0$

$$
\|B(u, v)\|_{V^{\prime}} \leq C_{3}|u||v|, \quad \forall u, v \in H .
$$

(iii) $\quad B: H \times D(A) \rightarrow V$ is a bounded bilinear operator and for a constant $C_{4}>0$

$$
\|B(u, v)\|_{V} \leq C_{4}|u||A v|, \quad \forall u \in H, v \in D(A) .
$$

(iv) For every $u \in V$ and $v \in H$

$$
(B(u, v), v)=0 .
$$

We now present one more important property of the nonlinear operator $B$ in the following lemma which will play important role in the later part of this section. The proof is straightforward and uses the bilinearity property of $B$.

Lemma 3.4. If $w=u-v$, then

$$
B(u, u)-B(v, v)=B(v, w)+B(w, v)+B(w, w) .
$$

With above functional setting and following the classical treatment of the Navier-Stokes equation, and in order to simplify the notation one can write the stochastic GOY model of turbulence (3.1) in a Hilbert space $H$ in the following way,

$$
\begin{aligned}
& \mathrm{d} u+[\nu A u+B(u, u)] \mathrm{d} t=f(t) \mathrm{d} t+\sigma(t, u) \mathrm{d} W(t) \\
& u(0)=u_{0},
\end{aligned}
$$

where $u=\left(u_{1}, u_{2}, \ldots\right) \in H$, the operators $A$ and $B$ are defined through (3.3) and (3.5), respectively, $f=\left(f_{1}, f_{2}, \ldots\right), \sigma(t, u)=\left(\sigma_{1}\left(t, u_{1}\right), \sigma_{2}\left(t, u_{2}\right), \ldots\right)$, and $W=\left(w_{1}, w_{2}, \ldots\right)$. Here $\left(W(t)_{t \geq 0}\right)$ is a $H$-valued Wiener process with trace class covariance, and the space $L_{Q}$ has been defined in Sect. 2. The noise coefficient $\sigma:[0, T] \times V \rightarrow L_{Q}\left(H_{0} ; H\right)$ is such that it satisfies the following hypotheses:

A.1. The function $\sigma \in C\left([0, T] \times V ; L_{Q}\left(H_{0} ; H\right)\right)$

A.2. For all $t \in(0, T)$, there exists a positive constant $K$ such that $|\sigma(t, u)|_{L_{Q}}^{2} \leq$ $K\left(1+\|u\|^{2}\right)$.

A.3. For all $t \in(0, T)$, there exists a positive constant $L$ such that for all $u, v \in V$, $|\sigma(t, u)-\sigma(t, v)|_{L_{Q}}^{2} \leq L\|u-v\|^{2}$. 
Remark 3.5. The above hypotheses can be verified from the assumptions $(A .1-$ $A .2)$ on the noise coefficients. Notice that, $Q: H \rightarrow H$ is a trace class covariance (nuclear) operator and hence compact. So $H_{0}=Q^{1 / 2} H$ is a separable Hilbert space and the imbedding of $H_{0}$ in $H$ is Hilbert-Schmidt. Let $\left\{e_{n}\right\}_{n=1}^{\infty}$ be the eigenfunctions of $Q$ (may not be complete). Then $Q e_{n}=\lambda_{n} e_{n}$, where each $\lambda_{n}$ is positive real and $\sum_{n} \lambda_{n}<\infty$. Note,

$$
\begin{aligned}
|\sigma(t, u)|_{L_{Q}}^{2} & =\sum_{m, n=1}^{\infty}\left|\left(\sigma h_{m}, e_{n}\right)\right|^{2}=\sum_{m, n=1}^{\infty} \lambda_{m}\left|\left(\sigma e_{m}, e_{n}\right)\right|^{2} \\
& =\left(\sigma Q^{1 / 2}, \sigma Q^{1 / 2}\right)=\operatorname{Tr}(\sigma Q \sigma),
\end{aligned}
$$

where $\left\{h_{m}\right\}$, with $h_{m}=\sqrt{\lambda_{m}} e_{m}, m=1,2, \ldots$ are orthonormal basis in $H_{0}$. have

Then, using assumption $(A .1)$ and letting $\lambda=\sup _{1 \leq n<\infty} \lambda_{n}<\infty$, one can

$$
\begin{aligned}
|\sigma(t, u)|_{L_{Q}}^{2} & =\sum_{n=1}^{\infty} \lambda_{n}\left|\sigma_{n}\left(t, u_{n}\right)\right|^{2} \leq\left(\sup _{1 \leq n<\infty} \lambda_{n}\right) \sum_{n=1}^{\infty}\left|\sigma_{n}\left(t, u_{n}\right)\right|^{2} \\
& \leq K_{1} \lambda \sum_{n=1}^{\infty} k_{n}^{2}\left|u_{n}\right|^{2} \leq K\left(1+\|u\|_{V}^{2}\right),
\end{aligned}
$$

which shows that hypothesis $(A .2)$ holds.

Similarly,

$$
\begin{aligned}
|\sigma(t, u)-\sigma(t, v)|_{L_{Q}}^{2} & \leq \lambda \sum_{n=1}^{\infty}\left|\sigma_{n}\left(t, u_{n}\right)-\sigma_{n}\left(t, v_{n}\right)\right|^{2} \\
& \leq \lambda K_{2} \sum_{n=1}^{\infty} k_{n}^{2}\left|u_{n}-v_{n}\right|^{2}=L\|u-v\|_{V}^{2} .
\end{aligned}
$$

Thus the hypothesis (A.3.) holds true.

Thus in the abstract setting of stochastic GOY model, the assumptions (a.1a.2) are required on the noise coefficient to impose the hypotheses (A.1-A.3) in the Hilbert space valued construction.

In the following lemma, we will show that sum of the linear and nonlinear operator is locally monotone in the $l^{4}$-ball.

Lemma 3.6. For a given $r>0$, let us denote by $\mathbb{B}_{r}$ the closed $l^{4}$-ball in $V$ :

$$
\mathbb{B}_{r}=\left\{v \in V ;\|v\|_{l^{4}} \leq r\right\} .
$$

Define the nonlinear operator $F$ on $V$ by $F(u):=-\nu A u-B(u, u)$. Then for any $0<\varepsilon<\frac{\nu}{2 L}$, where $L$ is the positive constant that appears in the condition (A.3), the pair $(F, \sqrt{\varepsilon} \sigma)$ is monotone in $\mathbb{B}_{r}$, i.e. for any $u \in V$ and $v \in \mathbb{B}_{r}$

$$
(F(u)-F(v), w)-\frac{r^{4}}{\nu^{3}}|w|^{2}+\varepsilon|\sigma(t, u)-\sigma(t, v)|_{L_{Q}}^{2} \leq 0,
$$

where $w=u-v$. 
Proof. First note that,

$$
\nu(A w, w)=\nu\|w\|^{2}
$$

Next using the Lemma 3.4 and Eq. (3.10) from Lemma 3.3, we have

$$
(B(u, u)-B(v, v), w)=(B(v, w)+B(w, v)+B(w, w), w)=(B(w, v), w) .
$$

Now using the definition of the operator $B$ and Eq. (3.4) from Lemma 3.2, we get for $C>0$,

$$
\begin{aligned}
|(B(w, v), w)|= & \mid \sum_{n=1}^{\infty} i k_{n}\left[\frac{1}{4} v_{n-1}^{\star} w_{n+1}^{\star} w_{n}^{\star}-\frac{1}{2}\left(w_{n+1}^{\star} v_{n+2}^{\star}+w_{n+2}^{\star} v_{n+1}^{\star}\right) w_{n}^{\star}\right. \\
& \left.+\frac{1}{8} w_{n-1}^{\star} v_{n-2}^{\star} w_{n}^{\star}\right] \mid \\
\leq & C\|v\|_{l^{4}}\|w\|_{l^{4}}\|w\| \\
\leq & \|v\|_{l^{4}}|w|^{1 / 2}\|w\|^{3 / 2} \\
\leq & \frac{\nu}{2}\|w\|^{2}+\frac{27}{32 \nu^{3}}|w|^{2}\|v\|_{l^{4}}^{4} .
\end{aligned}
$$

Since $v \in \mathbb{B}_{r}$, the above relation yields

$$
-(B(w, v), w) \leq \frac{\nu}{2}\|w\|^{2}+\frac{r^{4}}{\nu^{3}}|w|^{2} .
$$

Hence by the definition of the operator $F$,

$$
(F(u)-F(v), w) \leq-\frac{\nu}{2}\|w\|^{2}+\frac{r^{4}}{\nu^{3}}|w|^{2} .
$$

Finally, using condition (A.3) and that $\varepsilon<\frac{\nu}{2 L}$ we get the desired result.

Remark 3.7. Notice that the trilinear operator can also be estimated as follows,

$$
\begin{aligned}
|(B(w, v), w)| & \leq C|v||w|\left(\sup _{1 \leq n<\infty} k_{n}\left|w_{n}\right|\right) \\
& \leq C|v||w|\|w\|_{W^{1, \infty}} \\
& \leq C|v||w|\|w\|_{V}
\end{aligned}
$$

as it is obvious that,

$$
\|w\|_{W^{1, \infty}}=\left\|A^{1 / 2} w\right\|_{\infty} \leq\left\|A^{1 / 2} w\right\|_{2}=\|w\|_{V} .
$$

Then

$$
|(B(w, v), w)| \leq \frac{\nu}{2}\|w\|^{2}+\frac{1}{2 \nu}|w|^{2}|v|^{2} .
$$

Proceeding as in the previous Lemma one can see that the operator $F(u)=$ $-\nu A u-B(u, u)$ is also locally monotone in $l^{2}$-ball. But in this paper we prefer to work with $l^{4}$-local monotonicity, since it is better as the norm is weaker. 


\subsection{Energy estimate and existence theory}

Let $H_{n}:=\operatorname{span}\left\{e_{1}, e_{2}, \ldots, e_{n}\right\}$ where $\left\{e_{j}\right\}$ is any fixed orthonormal basis in $H$ with each $e_{j} \in D(A)$. Let $P_{n}$ denote the orthogonal projection of $H$ to $H_{n}$. Define $u^{n}=P_{n} u$, not to cause any confusion in notation with earlier $u_{n}$. Let $W_{n}=P_{n} W$. Let $\sigma_{n}=P_{n} \sigma$. Define $u^{n, \varepsilon}$ as the solution of the following stochastic differential equation in the variational form such that for each $v \in H_{n}$,

$$
\mathrm{d}\left(u^{n, \varepsilon}(t), v\right)=\left(F\left(u^{n, \varepsilon}(t)\right), v\right) \mathrm{d} t+(f(t), v) \mathrm{d} t+\sqrt{\varepsilon}\left(\sigma_{n}\left(t, u^{n, \varepsilon}(t)\right) \mathrm{d} W_{n}(t), v\right),
$$

with $u^{n, \varepsilon}(0)=P_{n} u(0)$.

The standard theory of finite-dimensional stochastic differential equations [21] guarantees the existence of a unique solution to (3.15) under the assumptions (A.1-A.3) if $f \in L^{2}([0, T], H)$ and $u(0)$ is $\mathcal{F}_{0}$ measurable and in $L^{2}(P)$.

Theorem 3.8. Under the above mathematical setting let $f$ be in $\mathrm{L}^{2}([0, T], H), u(0)$ be $\mathcal{F}_{0}$ measurable and $E|u(0)|^{2}<\infty$. Let $u^{n, \varepsilon}$ denote the unique strong solution of the stochastic differential equation (3.15) in $C\left([0, T], H_{n}\right)$. Then with $K$ as in condition (A.2), the following estimates hold:

For all $\varepsilon<\frac{\nu}{2 K}$, and $0 \leq t \leq T$,

$$
E\left|u^{n, \varepsilon}(t)\right|^{2}+\frac{\nu}{2} \int_{0}^{t} E\left\|u^{n, \varepsilon}(s)\right\|^{2} \mathrm{~d} s \leq E|u(0)|^{2}+\frac{1}{\nu} \int_{0}^{t}\|f(s)\|_{V^{\prime}}^{2} \mathrm{~d} s+\varepsilon K T,
$$

and for all $\varepsilon<\frac{1}{2 K^{2}} \wedge\left(\sqrt{\frac{\nu}{K}+\frac{1}{2}}-\frac{1}{\sqrt{2}}\right)^{2}$

$E\left[\sup _{0 \leq t \leq T}\left|u^{n, \varepsilon}(t)\right|^{2}+\frac{\nu}{2} \int_{0}^{T}\left\|u^{n, \varepsilon}(t)\right\|^{2} \mathrm{~d} t\right] \leq C\left(E|u(0)|^{2}, \int_{0}^{T}\|f(t)\|_{V^{\prime}}^{2} \mathrm{~d} t, \nu, T\right)$.

Also, for any $\delta>0$ and $\varepsilon<\frac{3 \nu}{2 K}$,

$$
\begin{aligned}
& E\left|u^{n, \varepsilon}(t)\right|^{2} e^{-\delta t}+\frac{\nu}{2} \int_{0}^{T} E\left\|u^{n, \varepsilon}(t)\right\|^{2} e^{-\delta t} \mathrm{~d} t \leq E|u(0)|^{2} \\
& +\frac{1}{\delta} \int_{0}^{T}|f(t)|^{2} e^{-\delta t} \mathrm{~d} t+\frac{\varepsilon K}{\delta}
\end{aligned}
$$

Moreover, if we suppose that $f \in \mathrm{L}^{4}([0, T], H)$ and $E|u(0)|^{4}<\infty$, then for all $\varepsilon<\frac{\nu}{3 K}$ and $0 \leq t \leq T$,

$$
\begin{aligned}
& E\left[\sup _{0 \leq t \leq T}\left|u^{n, \varepsilon}(t)\right|^{4} e^{-\delta t}+C_{\nu} \int_{0}^{T}\left\|u^{n, \varepsilon}(t)\right\|^{2}\left|u^{n, \varepsilon}(t)\right|^{2} e^{-\delta t} \mathrm{~d} t\right] \\
& \leq E|u(0)|^{4}+C_{\delta, T} \int_{0}^{T}\|f(t)\|_{V^{\prime}}^{4} e^{-\delta t} \mathrm{~d} t+\frac{\varepsilon M}{\delta} .
\end{aligned}
$$


Proof. Replacing $v$ with $u^{n, \varepsilon}$ in (3.15) and using the properties of the operators $A$ and $B$, we notice that,

$$
\begin{aligned}
& \mathrm{d}\left|u^{n, \varepsilon}(t)\right|^{2}+2 \nu\left\|u^{n, \varepsilon}(t)\right\|^{2} \mathrm{~d} t \\
&= 2\left(f(t), u^{n, \varepsilon}(t)\right) \mathrm{d} t+\varepsilon \operatorname{Tr}\left(\sigma_{n}\left(t, u^{n, \varepsilon}(t)\right) Q \sigma_{n}\left(t, u^{n, \varepsilon}(t)\right)\right) \mathrm{d} t \\
&+2 \sqrt{\varepsilon}\left(\sigma_{n}\left(t, u^{n, \varepsilon}(t)\right), u^{n, \varepsilon}(t)\right) \mathrm{d} W_{n}(t) .
\end{aligned}
$$

Using the inequality

$$
2 a b \leq \delta a^{2}+\frac{1}{\delta} b^{2}
$$

on $2\left(f(t), u^{n, \varepsilon}(t)\right)$ and using the condition (A.2), we obtain

$$
\begin{aligned}
& \mathrm{d}\left|u^{n, \varepsilon}(t)\right|^{2}+2 \nu\left\|u^{n, \varepsilon}(t)\right\|^{2} \mathrm{~d} t \leq\left(\nu\left\|u^{n, \varepsilon}(t)\right\|^{2}+\frac{1}{\nu}\|f(t)\|_{V^{\prime}}^{2}\right) \mathrm{d} t \\
& \quad+\varepsilon K\left(1+\left\|u^{n, \varepsilon}(t)\right\|^{2}\right) \mathrm{d} t+2 \sqrt{\varepsilon}\left(\sigma_{n}\left(t, u^{n, \varepsilon}(t)\right), u^{n, \varepsilon}(t)\right) \mathrm{d} W_{n}(t) .
\end{aligned}
$$

Define

$$
\tau_{N}=\inf \left\{t:\left|u^{n, \varepsilon}(t)\right|^{2}+\int_{0}^{t} \mid u^{n, \varepsilon}(s) \|^{2} \mathrm{~d} s>N\right\}
$$

Then integrating one can deduce

$$
\begin{aligned}
& \left|u^{n, \varepsilon}\left(t \wedge \tau_{N}\right)\right|^{2}+(\nu-\varepsilon K) \int_{0}^{t \wedge \tau_{N}}\left\|u^{n, \varepsilon}(s)\right\|^{2} \mathrm{~d} s \\
& \leq|u(0)|^{2}+\frac{1}{\nu} \int_{0}^{t \wedge \tau_{N}}\|f(s)\|_{V^{\prime}}^{2} \mathrm{~d} s+\varepsilon K \int_{0}^{t \wedge \tau_{N}} \mathrm{~d} s \\
& \quad+2 \sqrt{\varepsilon} \int_{0}^{t \wedge \tau_{N}}\left(\sigma_{n}\left(s, u^{n, \varepsilon}(s)\right), u^{n, \varepsilon}(s)\right) \mathrm{d} W_{n}(s) .
\end{aligned}
$$

Let $\varepsilon<\frac{\nu}{2 K}$. Then taking expectation on both sides, and using the fact that the the stochastic integral appeared in the last term of right hand side in the above estimate is a martingale, and hence has a zero average, we get

$$
\begin{gathered}
E\left[\left|u^{n, \varepsilon}\left(t \wedge \tau_{N}\right)\right|^{2}+(\nu-\varepsilon K) \int_{0}^{t \wedge \tau_{N}}\left\|u^{n, \varepsilon}(s)\right\|^{2} \mathrm{~d} s\right] \\
\leq E|u(0)|^{2}+\frac{1}{\nu} \int_{0}^{t \wedge \tau_{N}}\|f(s)\|_{V^{\prime}}^{2} \mathrm{~d} s+\varepsilon K T,
\end{gathered}
$$

thus we have the result (3.16). 
To prove (3.17), we proceed in the similar way as above, but we take supremum upto time $T \wedge \tau_{N}$ before taking the expectation,

$$
\begin{aligned}
E & {\left[\sup _{0 \leq t \leq T \wedge \tau_{N}}\left|u^{n, \varepsilon}(t)\right|^{2}+(\nu-\varepsilon K) \int_{0}^{T \wedge \tau_{N}}\left\|u^{n, \varepsilon}(t)\right\|^{2} \mathrm{~d} t\right] } \\
\leq & E|u(0)|^{2}+\frac{1}{\nu} \int_{0}^{T \wedge \tau_{N}}\|f(t)\|_{V^{\prime}}^{2} \mathrm{~d} t+\varepsilon K T \\
& +2 \sqrt{\varepsilon} E\left[\sup _{0 \leq t \leq T \wedge \tau_{N}}\left|\int_{0}^{t}\left(\sigma_{n}\left(s, u^{n, \varepsilon}(s)\right), u^{n, \varepsilon}(s)\right) \mathrm{d} W_{n}(s)\right|\right] .
\end{aligned}
$$

By means of Burkholder-Davis-Gundy inequality, condition (A.2) and CauchySchwartz inequality,

$$
\begin{aligned}
& 2 \sqrt{\varepsilon} E\left[\sup _{0 \leq t \leq T \wedge \tau_{N}}\left|\int_{0}^{t}\left(\sigma_{n}\left(s, u^{n, \varepsilon}(s)\right), u^{n, \varepsilon}(s)\right) \mathrm{d} W_{n}(s)\right|\right] \\
& \leq 2 \sqrt{2 \varepsilon} K E\left[\left(\int_{0}^{T \wedge \tau_{N}}\left(1+\left\|u^{n, \varepsilon}(t)\right\|^{2}\right)\left|u^{n, \varepsilon}(t)\right|^{2} \mathrm{~d} t\right)^{1 / 2}\right] \\
& \leq 2 \sqrt{2 \varepsilon} K E\left[\sup _{0 \leq t \leq T \wedge \tau_{N}}\left|u^{n, \varepsilon}(t)\right|\left(\int_{0}^{T \wedge \tau_{N}}\left(1+\left\|u^{n, \varepsilon}(t)\right\|^{2}\right) \mathrm{d} t\right)^{1 / 2}\right] \\
& \quad \leq \sqrt{2 \varepsilon} K\left[E\left(\sup _{0 \leq t \leq T \wedge \tau_{N}}\left|u^{n, \varepsilon}(t)\right|^{2}\right)+E \int_{0}^{T \wedge \tau_{N}}\left\|u^{n, \varepsilon}(t)\right\|^{2} \mathrm{~d} t+T\right] .
\end{aligned}
$$

Replace (3.21) in (3.20),

$$
\begin{aligned}
& (1-\sqrt{2 \varepsilon} K) E\left[\sup _{0 \leq t \leq T \wedge \tau_{N}}\left|u^{n, \varepsilon}(t)\right|^{2}\right]+(\nu-\varepsilon K-\sqrt{2 \varepsilon} K) \int_{0}^{T \wedge \tau_{N}}\left\|u^{n, \varepsilon}(t)\right\|^{2} \mathrm{~d} t \\
& \quad \leq E|u(0)|^{2}+\frac{1}{\nu} \int_{0}^{T \wedge \tau_{N}}\|f(t)\|_{V^{\prime}}^{2} \mathrm{~d} t+K T(\varepsilon+\sqrt{2 \varepsilon}) .
\end{aligned}
$$

Let

$$
\varepsilon<\frac{1}{2 K^{2}} \wedge\left(\sqrt{\frac{\nu}{K}+\frac{1}{2}}-\frac{1}{\sqrt{2}}\right)^{2} .
$$

Note $T \wedge \tau_{N} \rightarrow T$ a.s. as $N \rightarrow \infty$. Thus taking the limit in the above estimate (3.22) as $N \rightarrow \infty$, one can get the desired energy estimate (3.17).

Next, we consider the function $e^{-\delta t}\left|u^{n, \varepsilon}(t)\right|^{2}$ for $\delta>0$ and apply the Itô Lemma to get,

$$
\begin{aligned}
\mathrm{d}[ & \left.\left|u^{n, \varepsilon}(t)\right|^{2} e^{-\delta t}\right]+2 \nu\left\|u^{n, \varepsilon}(t)\right\|^{2} e^{-\delta t} \mathrm{~d} t+\delta\left|u^{n, \varepsilon}(t)\right|^{2} e^{-\delta t} \mathrm{~d} t \\
= & {\left[2\left(f(t), u^{n, \varepsilon}(t)\right)+\varepsilon \operatorname{Tr}\left(\sigma_{n}\left(t, u^{n, \varepsilon}(t)\right) Q \sigma_{n}\left(t, u^{n, \varepsilon}(t)\right)\right)\right] e^{-\delta t} \mathrm{~d} t } \\
& +2 \sqrt{\varepsilon}\left(\sigma_{n}\left(t, u^{n, \varepsilon}(t)\right), u^{n, \varepsilon}(t)\right) e^{-\delta t} \mathrm{~d} W_{n}(t) .
\end{aligned}
$$


Note that

$$
2\left(f(t), u^{n, \varepsilon}(t)\right) \leq \delta\left|u^{n, \varepsilon}(t)\right|^{2}+\frac{1}{\delta}|f(t)|^{2} .
$$

Hence upon writing (3.23) in the integral form, taking expectation and using condition (A.2), one can get

$$
\begin{aligned}
& E\left|u^{n, \varepsilon}(t)\right|^{2} e^{-\delta t}+2 \nu E \int_{0}^{T}\left\|u^{n, \varepsilon}(t)\right\|^{2} e^{-\delta t} \mathrm{~d} t \\
& \leq E|u(0)|^{2}+\frac{1}{\delta} \int_{0}^{T}|f(t)|^{2} e^{-\delta t} \mathrm{~d} t+\varepsilon K E \int_{0}^{T}\left(1+\left\|u^{n, \varepsilon}(t)\right\|^{2} e^{-\delta t} \mathrm{~d} t,\right.
\end{aligned}
$$

which yields the estimate (3.18) for all $\varepsilon<\frac{3 \nu}{2 K}$.

To prove (3.19), we first apply Itô Lemma on the function $\left|u^{n, \varepsilon}(t)\right|^{4} e^{-\delta t}$ to get,

$$
\begin{aligned}
\mathrm{d}[ & {\left[\left|u^{n, \varepsilon}(t)\right|^{4} e^{-\delta t}\right]+4 \nu\left\|u^{n, \varepsilon}(t)\right\|^{2}\left|u^{n, \varepsilon}(t)\right|^{2} e^{-\delta t} \mathrm{~d} t+\delta\left|u^{n, \varepsilon}(t)\right|^{4} e^{-\delta t} \mathrm{~d} t } \\
= & \left|u^{n, \varepsilon}(t)\right|^{2}\left[4\left(f(t), u^{n, \varepsilon}(t)\right)+8 \varepsilon \operatorname{Tr}\left(\sigma_{n}\left(t, u^{n, \varepsilon}(t)\right) Q \sigma_{n}\left(t, u^{n, \varepsilon}(t)\right)\right)\right] e^{-\delta t} \mathrm{~d} t \\
& +4 \sqrt{\varepsilon}\left(\sigma_{n}\left(t, u^{n, \varepsilon}(t)\right), u^{n, \varepsilon}(t)\right)\left|u^{n, \varepsilon}(t)\right|^{2} e^{-\delta t} \mathrm{~d} W_{n}(t) .
\end{aligned}
$$

Using the fact that

$$
4\left(f(t), u^{n, \varepsilon}(t)\right)\left|u^{n, \varepsilon}(t)\right|^{2} \leq C_{\delta}|f(t)|^{4}+\delta\left|u^{n, \varepsilon}(t)\right|^{4},
$$

and applying the condition (A.2) and integrating we have,

$$
\begin{aligned}
& \left|u^{n, \varepsilon}(t)\right|^{4} e^{-\delta t}+(4 \nu-8 \varepsilon K) \int_{0}^{t}\left\|u^{n, \varepsilon}(s)\right\|^{2}\left|u^{n, \varepsilon}(s)\right|^{2} e^{-\delta s} \mathrm{~d} s \\
& \leq E|u(0)|^{4}+C_{\delta} \int_{0}^{t}|f(s)|^{4} e^{-\delta s} \mathrm{~d} s \\
& \quad+4 \sqrt{\varepsilon} \int_{0}^{t}\left(\sigma_{n}\left(s, u^{n, \varepsilon}(s)\right), u^{n, \varepsilon}(s)\right)\left|u^{n, \varepsilon}(s)\right|^{2} e^{-\delta s} \mathrm{~d} W_{n}(s) .
\end{aligned}
$$

Finally, taking supremum in $0 \leq t \leq T$, then taking expectation on both sides and using the Burkholder-Davis-Gundy inequality on the stochastic integral term, we get the estimate (3.19).

Definition 3.9. (Strong Solution) A strong solution $u^{\varepsilon}$ is defined on a given probability space $\left(\Omega, \mathcal{F}, \mathcal{F}_{t}, P\right)$ as a $\mathrm{L}^{2}\left(\Omega ; \mathrm{L}^{\infty}(0, T ; H) \cap \mathrm{L}^{2}(0, T ; V) \cap C(0, T ; H)\right)$ valued adapted process which satisfies the stochastic GOY model

$$
\begin{aligned}
& \mathrm{d} u^{\varepsilon}+\left[\nu A u^{\varepsilon}+B\left(u^{\varepsilon}, u^{\varepsilon}\right)\right] \mathrm{d} t=f(t) \mathrm{d} t+\sqrt{\varepsilon} \sigma\left(t, u^{\varepsilon}\right) \mathrm{d} W(t) \\
& u^{\varepsilon}(0)=u_{0},
\end{aligned}
$$

in the weak sense and also the energy inequalities in Theorem 3.8.

Monotonicty arguments were first used by Krylov and Rozovskii [22] to prove the existence and uniqueness of the strong solutions for a wide class of stochastic evolution equations (under certain assumptions on the drift and diffusion coefficients), which in fact is the refinement of the previous results by Pardoux $[30,31]$ 
and also the generalization of the results by Bensoussan and Temam [3]. Menaldi and Sritharan [27] further developed this theory for the case when the sum of the linear and nonlinear operators are locally monotone.

Theorem 3.10. Let $u(0)$ be $\mathcal{F}_{0}$ measurable and $E\left|u_{0}\right|^{4}<\infty$. Let $f \in \mathrm{L}^{4}\left(0, T ; V^{\prime}\right)$. We also assume that $0<\varepsilon<\frac{\nu}{L}$ and the diffusion coefficient satisfies the conditions (A.1-A.3). Then there exists a unique adapted process $u^{\varepsilon}(t, x, w)$ with the regularity

$$
u^{\varepsilon} \in \mathrm{L}^{2}\left(\Omega ; C(0, T ; H) \cap \mathrm{L}^{2}(0, T ; V)\right)
$$

satisfying the stochastic GOY model (3.25) and the a priori bounds in Theorem 3.8.

Proof. Part I. (Existence)

Using the a priori estimate in the Theorem 3.8, it follows from the Banach-Alaoglu theorem that along a subsequence, the Galerkin approximations $\left\{u^{n, \varepsilon}\right\}$ have the following limits:

$$
\begin{aligned}
& u^{n, \varepsilon} \longrightarrow u^{\varepsilon} \quad \text { weak star in } \mathrm{L}^{4}\left(\Omega ; \mathrm{L}^{\infty}(0, T ; H)\right) \cap \mathrm{L}^{2}\left(\Omega ; \mathrm{L}^{2}(0, T ; V)\right), \\
& F\left(u^{n, \varepsilon}\right) \longrightarrow F_{0}^{\varepsilon} \quad \text { weakly } \operatorname{inL}^{2}\left(\Omega ; \mathrm{L}^{2}\left(0, T ; V^{\prime}\right)\right), \\
& \sigma_{n}\left(\cdot, u^{n, \varepsilon}\right) \longrightarrow S^{\varepsilon} \quad \text { weakly in } \mathrm{L}^{2}\left(\Omega ; \mathrm{L}^{2}\left(0, T ; \mathrm{L}_{Q}\right)\right) .
\end{aligned}
$$

The assertion of the second statement holds since $F\left(u^{n, \varepsilon}\right)$ is bounded in $\mathrm{L}^{2}(\Omega$; $\left.\mathrm{L}^{2}\left(0, T ; V^{\prime}\right)\right)$. Likewise since diffusion coefficient has the linear growth property and $u^{n, \varepsilon}$ is bounded in $\mathrm{L}^{2}(0, T ; V)$ uniformly in $n$, the last statement holds. Then $u^{\varepsilon}$ has the Itô differential

$$
\mathrm{d} u^{\varepsilon}(t)=F_{0}^{\varepsilon}(t) \mathrm{d} t+\sqrt{\varepsilon} S^{\varepsilon}(t) \mathrm{d} W(t) \quad \text { weakly in } \mathrm{L}^{2}\left(\Omega ; \mathrm{L}^{2}\left(0, T ; V^{\prime}\right)\right) .
$$

Let us set,

$$
r(t):=\frac{2}{\nu^{3}} \int_{0}^{t}\left\|v^{\varepsilon}(s)\right\|_{\mathrm{L}^{4}}^{4} \mathrm{~d} s
$$

where $v^{\varepsilon}(t, x, \omega)$ is any adapted process in $\mathrm{L}^{\infty}(\Omega \times(0, T) ; H)$. Here we suppress the dependence of $\varepsilon$ in the notation of $r$ to make it easier to read. Then applying the Itô Lemma to the function $2 e^{-r(t)}\left|u^{n, \varepsilon}(t)\right|^{2}$, one obtains

$$
\begin{aligned}
\mathrm{d}\left[e^{-r(t)}\left|u^{n, \varepsilon}(t)\right|^{2}\right]= & e^{-r(t)}\left(2 F\left(u^{n, \varepsilon}(t)\right)-\dot{r}(t) u^{n, \varepsilon}(t), u^{n, \varepsilon}(t)\right) \mathrm{d} t \\
& +\varepsilon e^{-r(t)}\left|\sigma_{n}\left(t, u^{n, \varepsilon}(t)\right)\right|_{\mathrm{L}_{Q}}^{2} \mathrm{~d} t \\
& +2 \sqrt{\varepsilon} e^{-r(t)}\left(\sigma_{n}\left(t, u^{n, \varepsilon}(t)\right), u^{n, \varepsilon}(t)\right) \mathrm{d} W(t) .
\end{aligned}
$$


Integrating between $0 \leq t \leq T$ and taking expectation,

$$
\begin{aligned}
& E\left[e^{-r(T)}\left|u^{n, \varepsilon}(T)\right|^{2}-\left|u^{n, \varepsilon}(0)\right|^{2}\right] \\
& =E\left[\int_{0}^{T} e^{-r(t)}\left(2 F\left(u^{n, \varepsilon}(t)\right)-\dot{r}(t) u^{n, \varepsilon}(t), u^{n, \varepsilon}(t)\right) \mathrm{d} t\right] \\
& \quad+\varepsilon E \int_{0}^{T} e^{-r(t)}\left|\sigma_{n}\left(t, u^{n, \varepsilon}(t)\right)\right|_{\mathrm{L}_{Q}}^{2} \mathrm{~d} t \\
& \quad+2 \sqrt{\varepsilon} E \int_{0}^{T} e^{-r(t)}\left(\sigma_{n}\left(t, u^{n, \varepsilon}(t)\right), u^{n, \varepsilon}(t)\right) \mathrm{d} W(t) .
\end{aligned}
$$

The last term on the right hand side vanishes since the integral inside the expectation is a martingale. Then by the lower semi-continuity property of the weak convergence,

$$
\begin{aligned}
& \liminf _{n} E\left[\int_{0}^{T} e^{-r(t)}\left(2 F\left(u^{n, \varepsilon}(t)\right)-\dot{r}(t) u^{n, \varepsilon}(t), u^{n, \varepsilon}(t)\right) \mathrm{d} t\right. \\
& \left.\quad+\varepsilon \int_{0}^{T} e^{-r(t)}\left|\sigma_{n}\left(t, u^{n, \varepsilon}(t)\right)\right|_{\mathrm{L}_{Q}}^{2} \mathrm{~d} t\right] \\
& =\liminf _{n} E\left[e^{-r(T)}\left|u^{n, \varepsilon}(T)\right|^{2}-\left|u^{n, \varepsilon}(0)\right|^{2}\right] \\
& \geq E\left[e^{-r(T)}\left|u^{\varepsilon}(T)\right|^{2}-\left|u^{\varepsilon}(0)\right|^{2}\right] \\
& =E\left[\int_{0}^{T} e^{-r(t)}\left(2 F_{0}^{\varepsilon}(t)-\dot{r}(t) u^{\varepsilon}(t), u^{\varepsilon}(t)\right) \mathrm{d} t+\varepsilon \int_{0}^{T} e^{-r(t)}\left|S^{\varepsilon}\right|_{\mathrm{L}_{Q}}^{2} \mathrm{~d} t\right] .
\end{aligned}
$$

Now by monotonicity property from Lemma 3.6,

$$
\begin{aligned}
& 2 E\left[\int_{0}^{T} e^{-r(t)}\left(F\left(u^{n, \varepsilon}(t)\right)-F\left(v^{\varepsilon}(t)\right), u^{n, \varepsilon}(t)-v^{\varepsilon}(t)\right) \mathrm{d} t\right] \\
& -E\left[\int_{0}^{T} e^{-r(t)} \dot{r}(t)\left|u^{n, \varepsilon}(t)-v^{\varepsilon}(t)\right|^{2} \mathrm{~d} t\right] \\
& \quad+\varepsilon E\left[\int_{0}^{T} e^{-r(t)}\left|\sigma_{n}\left(t, u^{n, \varepsilon}(t)\right)-\sigma_{n}\left(t, v^{\varepsilon}(t)\right)\right|_{\mathrm{L}_{Q}}^{2} \mathrm{~d} t\right] \\
& \leq 0 .
\end{aligned}
$$


Rearranging the terms,

$$
\begin{aligned}
& E\left[\int_{0}^{T} e^{-r(t)}\left(2 F\left(u^{n, \varepsilon}(t)\right)-\dot{r}(t) u^{n, \varepsilon}(t), u^{n, \varepsilon}(t)\right) \mathrm{d} t\right. \\
& \left.\quad+\varepsilon \int_{0}^{T} e^{-r(t)}\left|\sigma_{n}\left(t, u^{n, \varepsilon}(t)\right)\right|_{\mathrm{L}_{Q}}^{2} \mathrm{~d} t\right] \\
& \leq E\left[\int_{0}^{T} e^{-r(t)}\left(2 F\left(u^{n, \varepsilon}(t)\right)-\dot{r}(t)\left(2 u^{n, \varepsilon}(t)-v^{\varepsilon}(t)\right), v^{\varepsilon}(t)\right) \mathrm{d} t\right] \\
& +E\left[\int_{0}^{T} e^{-r(t)}\left(2 F\left(v^{\varepsilon}(t)\right), u^{n, \varepsilon}(t)-v^{\varepsilon}(t)\right) \mathrm{d} t\right] \\
& +\varepsilon E\left[\int_{0}^{T} e^{-r(t)}\left(2 \sigma_{n}\left(t, u^{n, \varepsilon}(t)\right)-\sigma_{n}\left(t, v^{\varepsilon}(t)\right), \sigma_{n}\left(t, v^{\varepsilon}(t)\right)\right)_{\mathrm{L}_{Q}} \mathrm{~d} t\right] .
\end{aligned}
$$

Taking limit in $n$, using the result from (3.28) and rearranging, we obtain

$$
\begin{aligned}
& E\left[\int_{0}^{T} e^{-r(t)}\left(2 F_{0}^{\varepsilon}(t)-2 F\left(v^{\varepsilon}(t)\right), u^{\varepsilon}(t)-v^{\varepsilon}(t)\right) \mathrm{d} t\right] \\
& \quad+E\left[\int_{0}^{T} e^{-r(t)} \dot{r}(t)\left|u^{\varepsilon}(t)-v^{\varepsilon}(t)\right|^{2} \mathrm{~d} t\right] \\
& \quad+\varepsilon E\left[\int_{0}^{T} e^{-r(t)}\left\|S(t)-\sigma\left(t, v^{\varepsilon}(t)\right)\right\|_{\mathrm{L}_{Q}}^{2} \mathrm{~d} t\right] \\
& \leq 0 .
\end{aligned}
$$

Notice that for $v^{\varepsilon}=u^{\varepsilon}, S(t)=\sigma\left(t, u^{\varepsilon}(t)\right)$. Take $v^{\varepsilon}=u^{\varepsilon}-\lambda w^{\varepsilon}$ with $\lambda>0$ and $w^{\varepsilon}$ is an adapted process in $\mathrm{L}^{2}\left(\Omega ; C(0, T ; H) \cap \mathrm{L}^{2}(0, T ; V)\right)$ Then,

$$
\begin{aligned}
& \lambda E\left[\int_{0}^{T} e^{-r(t)}\left(2 F_{0}^{\varepsilon}(t)-2 F\left(u^{\varepsilon}-\lambda w^{\varepsilon}\right)(t), w^{\varepsilon}(t)\right) \mathrm{d} t+\lambda \int_{0}^{T} e^{-r(t)} \dot{r}(t)\left|w^{\varepsilon}(t)\right|^{2} \mathrm{~d} t\right] \\
& \quad \leq 0 .
\end{aligned}
$$

Dividing by $\lambda$ on both sides of the inequality above and letting $\lambda$ go to 0 , one obtains

$$
E\left[\int_{0}^{T} e^{-r(t)}\left(F_{0}^{\varepsilon}(t)-F\left(u^{\varepsilon}(t)\right), w^{\varepsilon}(t)\right) \mathrm{d} t\right] \leq 0 .
$$

Since $w^{\varepsilon}$ is arbitrary, we conclude that $F_{0}^{\varepsilon}(t)=F\left(u^{\varepsilon}(t)\right)$. Thus the existence of the strong solution of the stochastic GOY model (3.25) has been proved. 
Part II. (Uniqueness)

If $v^{\varepsilon} \in \mathrm{L}^{2}\left(\Omega ; C(0, T ; H) \cap \mathrm{L}^{2}(0, T ; V)\right)$ be another solution of the equation $(3.25)$ then $w^{\varepsilon}=u^{\varepsilon}-v^{\varepsilon}$ solves the stochastic differential equation in $\mathrm{L}^{2}\left(\Omega ; \mathrm{L}^{2}\left(0, T ; V^{\prime}\right)\right)$,

$$
\mathrm{d} w^{\varepsilon}(t)=\left(F\left(u^{\varepsilon}(t)\right)-F\left(v^{\varepsilon}(t)\right)\right) \mathrm{d} t+\sqrt{\varepsilon}\left(\sigma\left(t, u^{\varepsilon}(t)\right)-\sigma\left(t, v^{\varepsilon}(t)\right)\right) \mathrm{d} W(t) .
$$

We denote $\sigma_{d}=\sigma\left(t, u^{\varepsilon}(t)\right)-\sigma\left(t, v^{\varepsilon}(t)\right)$.

We now apply Itô Lemma to the function $2 e^{-r(t)}\left|w^{\varepsilon}(t)\right|^{2}$ and using the local monotonicity of the sum of the linear and nonlinear operators $A$ and $B$, e.g. equation (3.14), we get

$$
\begin{aligned}
e^{-r(t)} \mathrm{d}\left|w^{\varepsilon}(t)\right|^{2}+\nu e^{-r(t)}\left\|w^{\varepsilon}(t)\right\|^{2} \mathrm{~d} t \leq & \frac{2 r^{4}}{\nu^{3}} e^{-r(t)}\left|w^{\varepsilon}(t)\right|^{2} \mathrm{~d} t+\varepsilon e^{-r(t)} \operatorname{Tr}\left(\sigma_{d} Q \sigma_{d}\right) \mathrm{d} t \\
& +2 \sqrt{\varepsilon} e^{-r(t)}\left(\sigma_{d}, w^{\varepsilon}(t)\right) \mathrm{d} W(t) .
\end{aligned}
$$

Using condition (A.3),

$$
\begin{aligned}
\mathrm{d}\left(e^{-r(t)}\left|w^{\varepsilon}(t)\right|^{2}\right)+\nu e^{-r(t)}\left\|w^{\varepsilon}(t)\right\|^{2} \mathrm{~d} t \leq & \varepsilon L e^{-r(t)}\left\|w^{\varepsilon}(t)\right\|^{2} \mathrm{~d} t \\
& +2 \sqrt{\varepsilon} e^{-r(t)}\left(\sigma_{d}, w^{\varepsilon}(t)\right) \mathrm{d} W(t) .
\end{aligned}
$$

Finally integrating in $0 \leq t \leq T$, taking expectation on both sides and noting $\varepsilon<\frac{\nu}{L}$ and the fact that

$$
\int_{0}^{T} e^{-r(t)}\left(\sigma_{d}, w^{\varepsilon}(t)\right) \mathrm{d} W(t)
$$

is a martingale for $T<\infty$, we obtain P-a.s.

$$
E\left[e^{-r(t)}\left|w^{\varepsilon}(t)\right|^{2}\right] \leq E|w(0)|^{2},
$$

which assures the uniqueness of the strong solution.

\section{Large deviation principle continued}

Let us recall the stochastic GOY model in consideration,

$$
\begin{aligned}
\mathrm{d} u^{\varepsilon}+\left[\nu A u^{\varepsilon}+B\left(u^{\varepsilon}, u^{\varepsilon}\right)\right] \mathrm{d} t & =f(t) \mathrm{d} t+\sqrt{\varepsilon} \sigma\left(t, u^{\varepsilon}\right) \mathrm{d} W(t) \\
u^{\varepsilon}(0) & =\xi,
\end{aligned}
$$

has a unique strong solution in the Polish space $X=C([0, T] ; H) \cap \mathrm{L}^{2}(0, T ; V)$. The solution to the stochastic GOY model, denoted by $u^{\varepsilon}$ can be written as $\mathcal{G}^{\varepsilon}(W(\cdot))$, for a Borel measurable function $\mathcal{G}^{\varepsilon}: C([0, T] ; H) \rightarrow X$ (see [21], page 310, [38], Chapter X, Corollary 4.2). The aim of this section is to verify that such a $\mathcal{G}^{\varepsilon}$ satisfies Assumption 2.10. Then applying the Theorem 2.11 the LDP for $\left\{u^{\varepsilon}: \varepsilon>0\right\}$ in $X$ can be established.

The LDP for $\left\{u^{\varepsilon}: \varepsilon>0\right\}$ in $X$ have been proved here systematically in four steps. In the first and second Theorems we show the well posedness of certain controlled stochastic and controlled deterministic equations in $X$. These results 
help to prove the last two main Theorems on the compactness of the level sets and weak convergence of the stochastic control equation stated in Assumption 2.10.

Theorem 4.1. For any $v \in \mathcal{A}_{M}, 0<M<\infty$, the stochastic control equation

$$
\begin{aligned}
& \mathrm{d} u_{v}^{\varepsilon}(t)+\left[\nu A u_{v}^{\varepsilon}(t)+B\left(u_{v}^{\varepsilon}(t), u_{v}^{\varepsilon}(t)\right)\right] \mathrm{d} t=\left[f(t)+\tilde{\sigma}\left(t, u_{v}^{\varepsilon}(t)\right) v(t)\right] \mathrm{d} t \\
& \quad+\sqrt{\varepsilon} \sigma\left(t, u_{v}^{\varepsilon}(t)\right) \mathrm{d} W(t), \\
& u_{v}^{\varepsilon}(0)=\xi \in H,
\end{aligned}
$$

has a unique strong solution in $\mathrm{L}^{2}(\Omega ; X)$, where $X=C(0, T ; H) \cap \mathrm{L}^{2}(0, T ; V)$, $f \in \mathrm{L}^{4}\left(0, T ; V^{\prime}\right)$ and $\sigma, \tilde{\sigma}$ both satisfy the hypotheses A.1-A.3. in Sect. 3.

Proof. We first prove that if $u_{v}^{\varepsilon}(t)$ is a strong solution of the stochastic controlled equation (4.2), the following energy estimate holds:

$$
E\left(\sup _{0 \leq t \leq T}\left|u_{v}^{\varepsilon}(t)\right|^{2}+\int_{0}^{T}\left\|u_{v}^{\varepsilon}(t)\right\|^{2} \mathrm{~d} t\right) \leq C,
$$

where

$$
C=C\left(|\xi|^{2}, \int_{0}^{T}\|f\|_{V^{\prime}}^{2} \mathrm{~d} t, \nu, K, T, M\right)
$$

is a positive constant.

To prove the above estimate, we apply Itô formula on $\left|u_{v}^{\varepsilon}(t)\right|^{2}$ and integrating in $0 \leq t \leq T$,

$$
\begin{aligned}
& \left|u_{v}^{\varepsilon}(t)\right|^{2}+\nu \int_{0}^{t}\left\|u_{v}^{\varepsilon}(s)\right\|^{2} \mathrm{~d} s \\
& \leq|\xi|^{2}+\frac{1}{\nu} \int_{0}^{t}\|f(s)\|_{V^{\prime}}^{2} \mathrm{~d} s+2 \int_{0}^{t}\left(\tilde{\sigma}\left(s, u_{v}^{\varepsilon}(s)\right) v(s), u_{v}^{\varepsilon}(s)\right) \mathrm{d} s \\
& \quad+\varepsilon \int_{0}^{t} \operatorname{Tr}\left(\sigma\left(s, u_{v}^{\varepsilon}(s)\right) Q \sigma\left(s, u_{v}^{\varepsilon}(s)\right)\right) \mathrm{d} s+2 \sqrt{\varepsilon} \int_{0}^{t}\left(\sigma\left(s, u_{v}^{\varepsilon}(s)\right), u_{v}^{\varepsilon}(s)\right) \mathrm{d} W(s) .
\end{aligned}
$$

Notice that,

$$
\begin{aligned}
& 2 \int_{0}^{t}\left(\tilde{\sigma}\left(s, u_{v}^{\varepsilon}(s)\right) v(s), u_{v}^{\varepsilon}(s)\right) \mathrm{d} s \\
& \quad \leq 2 \int_{0}^{t}\left|\tilde{\sigma}\left(s, u_{v}^{\varepsilon}(s)\right)\right|_{\mathrm{L}_{Q}}|v(s)|{ }_{0}\left|u_{v}^{\varepsilon}(s)\right| \mathrm{d} s \\
& \quad \leq \frac{\nu}{4 K} \int_{0}^{t}\left|\tilde{\sigma}\left(s, u_{v}^{\varepsilon}(s)\right)\right|_{\mathrm{L}_{Q}}^{2} \mathrm{~d} s+\frac{4 K}{\nu} \int_{0}^{t}|v(s)|_{0}^{2}\left|u_{v}^{\varepsilon}(s)\right|^{2} \mathrm{~d} s \\
& \quad \leq \frac{\nu}{4} \int_{0}^{t}\left(1+\left\|u_{v}^{\varepsilon}(s)\right\|^{2}\right) \mathrm{d} s+\frac{4 K}{\nu} \int_{0}^{t}|v(s)|_{0}^{2}\left|u_{v}^{\varepsilon}(s)\right|^{2} \mathrm{~d} s \\
& \quad \leq \frac{\nu}{4} \int_{0}^{t}\left(1+\left\|u_{v}^{\varepsilon}(s)\right\|^{2}\right) \mathrm{d} s+\frac{4 K M}{\nu} \sup _{0 \leq t \leq T}\left|u_{v}^{\varepsilon}(t)\right|^{2},
\end{aligned}
$$


and for $\varepsilon<\frac{\nu}{4 K}$,

$$
\varepsilon \int_{0}^{t} \operatorname{Tr}\left(\sigma\left(s, u_{v}^{\varepsilon}(s)\right) Q \sigma\left(s, u_{v}^{\varepsilon}(s)\right)\right) \mathrm{d} s \leq \frac{\nu}{4} \int_{0}^{t}\left(1+\left\|u_{v}^{\varepsilon}(s)\right\|^{2}\right) \mathrm{d} s .
$$

After rearrangement of the Eq. (4.4), we take supremum in time $0 \leq t \leq T$ and then expectation to get,

$$
\begin{aligned}
E & {\left[\sup _{0 \leq t \leq T}\left|u_{v}^{\varepsilon}(t)\right|^{2}+\frac{\nu}{2} \int_{0}^{T}\left\|u_{v}^{\varepsilon}(t)\right\|^{2} \mathrm{~d} t\right] } \\
\leq & |\xi|^{2}+\frac{\nu}{2} T+\frac{1}{\nu} \int_{0}^{T}\|f(t)\|_{V^{\prime}}^{2} \mathrm{~d} t+\frac{4 K M}{\nu} E\left[\sup _{0 \leq t \leq T}\left|u_{v}^{\varepsilon}(t)\right|^{2}\right] \\
& +2 \sqrt{\varepsilon} E\left[\sup _{0 \leq t \leq T}\left|\int_{0}^{t}\left(\sigma\left(s, u_{v}^{\varepsilon}(s)\right), u_{v}^{\varepsilon}(s)\right) \mathrm{d} W(s)\right|\right] .
\end{aligned}
$$

The last term of the above equation can be estimated in a similar manner as in (3.21),

$$
\begin{aligned}
& 2 \sqrt{\varepsilon} E\left[\sup _{0 \leq t \leq T}\left|\int_{0}^{t}\left(\sigma\left(s, u_{v}^{\varepsilon}(s)\right), u_{v}^{\varepsilon}(s)\right) \mathrm{d} W(s)\right|\right] \\
& \quad \leq \sqrt{2 \varepsilon} K\left[E\left(\sup _{0 \leq t \leq T}\left|u_{v}^{\varepsilon}(t)\right|^{2}\right)+E \int_{0}^{T}\left\|u_{v}^{\varepsilon}(t)\right\|^{2} \mathrm{~d} t+T\right] .
\end{aligned}
$$

Replacing (4.8) in (4.7), and considering

$$
\varepsilon<\min \left\{\frac{\nu}{4 K}, \frac{\nu^{2}}{8 K^{2}}, \frac{(1-4 K M / \nu)^{2}}{2 K^{2}}\right\}
$$

we get the energy estimate (4.3).

The proof of the existence and uniqueness of the strong solution of the stochastic controlled equation (4.2) follow from the Theorem 3.10, only a few modifications are needed due to the presence of the control term.

Theorem 4.2. Let $v \in \mathrm{L}^{2}\left(0, T ; H_{0}\right), f \in \mathrm{L}^{4}\left(0, T ; V^{\prime}\right)$ and $\sigma$ satisfy the hypotheses A.1.-A.3. in Sect. 3. Then the equation

$$
\mathrm{d} u_{v}(t)+\left[\nu A u_{v}(t)+B\left(u_{v}(t), u_{v}(t)\right)\right] \mathrm{d} t=f(t) \mathrm{d} t+\sigma\left(t, u_{v}(t)\right) v(t) \mathrm{d} t,
$$

where $u_{v}(0)=\xi \in H$, has a unique strong solution in $X=C(0, T ; H) \cap \mathrm{L}^{2}(0, T ; V)$.

Proof. This result can be considered as a particular case of the previous Theorem 4.1, where the diffusion coefficient is absent.

Next we state a important lemma from Budhiraja and Dupuis [4].

Lemma 4.3. Let $\left\{v_{n}\right\}$ be a sequence of elements from $\mathcal{A}_{M}$ for some finite $M>$ 0 . Let $v_{n}$ converges in distribution to $v$ with respect to the weak topology on $\mathrm{L}^{2}\left(0, T ; H_{0}\right)$. Then $\int_{0}^{\cdot} v_{n}(s) \mathrm{d} s$ converges in distribution as $C(0, T ; H)$-valued processes to $\int_{0}^{\cdot} v(s) \mathrm{d} s$ as $n \rightarrow \infty$.

Now we are ready to check the Assumptions 2.10. 
Theorem 4.4 (Compactness). Let $M<\infty$ be a fixed positive number. Let

$$
K_{M}:=\left\{u_{v} \in C(0, T ; H) \cap \mathrm{L}^{2}(0, T ; V) ; v \in S_{M}\right\},
$$

where $u_{v}$ is the unique solution in $X=C(0, T ; H) \cap \mathrm{L}^{2}(0, T ; V)$ of the deterministic controlled equation (4.9), with $u_{v}(0)=\xi \in H$. Then $K_{M}$ is compact in $X$.

Proof. Let us consider a sequence $\left\{u_{v_{n}}\right\}$ in $K_{M}$, where $u_{v_{n}}$ corresponds to the solution of (4.9) with control $v_{n} \in S_{M}$ in place of $v$, i.e.

$$
\mathrm{d} u_{v_{n}}(t)+\left[\nu A u_{v_{n}}(t)+B\left(u_{v_{n}}(t), u_{v_{n}}(t)\right)\right] \mathrm{d} t=f(t) \mathrm{d} t+\sigma\left(t, u_{v_{n}}(t)\right) v_{n}(t) \mathrm{d} t,
$$

with $u_{v_{n}}(0)=\xi \in H$. Then by weak compactness of $S_{M}$, there exists a subsequence of $\left\{v_{n}\right\}$, still denoted by $\left\{v_{n}\right\}$, which converges weakly to $v \in S_{M}$ in $\mathrm{L}^{2}\left(0, T ; H_{0}\right)$.

We need to prove $u_{v_{n}} \rightarrow u_{v}$ in $X$ as $n \rightarrow \infty$, or in other words,

$$
\sup _{0 \leq t \leq T}\left|u_{v_{n}}(t)-u_{v}(t)\right|^{2}+\int_{0}^{T}\left\|u_{v_{n}}(t)-u_{v}(t)\right\|^{2} \mathrm{~d} t \rightarrow 0,
$$

as $n \rightarrow \infty$.

According to the Theorem 4.2, $u_{v}$ is unique strong solution in $X$ of the deterministic controlled equation (4.9). Hence it is obvious to note that, $u_{v}$ satisfies the following a-priori estimate

$$
\sup _{0 \leq t \leq T}\left|u_{v}(t)\right|^{2}+\int_{0}^{T}\left\|u_{v}(t)\right\|^{2} \mathrm{~d} t \leq C
$$

where

$$
C=C\left(|\xi|^{2}, \int_{0}^{T}\|f\|_{V^{\prime}}^{2} \mathrm{~d} t, \nu, K, T, M\right)
$$

is a positive constant.

For the proof, we refer the Theorem 4.1, where the stochastic version of the above a priori estimate has been worked out.

Let $w_{v_{n}}=u_{v_{n}}-u_{v}$. Then $w_{v_{n}}$ satisfies the following differential equation

$$
\begin{aligned}
& \mathrm{d} w_{v_{n}}(t)+\left[\nu A w_{v_{n}}(t)+B\left(u_{v_{n}}(t), u_{v_{n}}(t)\right)-B\left(u_{v}(t), u_{v}(t)\right)\right] \mathrm{d} t \\
& \quad=\left[\sigma\left(t, u_{v_{n}}(t)\right) v_{n}(t)-\sigma\left(t, u_{v}(t)\right) v(t)\right] \mathrm{d} t,
\end{aligned}
$$

which yields

$$
\begin{aligned}
& \left|w_{v_{n}}(t)\right|^{2}+2 \nu \int_{0}^{t}\left\|w_{v_{n}}(s)\right\|^{2} \mathrm{~d} s \\
& \quad+2 \int_{0}^{t}\left(B\left(u_{v_{n}}(s), u_{v_{n}}(s)\right)-B\left(u_{v}(s), u_{v}(s)\right), w_{v_{n}}(s)\right) \mathrm{d} s \\
& =2 \int_{0}^{t}\left(\sigma\left(s, u_{v_{n}}(s)\right) v_{n}(s)-\sigma\left(s, u_{v}(s)\right) v(s), w_{v_{n}}(s)\right) \mathrm{d} s
\end{aligned}
$$


First note that, from Lemma 3.4,

$$
B\left(u_{v_{n}}, u_{v_{n}}\right)-B\left(u_{v}, u_{v}\right)=B\left(u_{v}, w_{v_{n}}\right)+B\left(w_{v_{n}}, u_{v}\right)+B\left(w_{v_{n}}, w_{v_{n}}\right) .
$$

Using the above expression and the properties $(i i)$ and $(i v)$ of the bilinear operator $B$ given in Lemma 3.3, one can find

$$
\begin{aligned}
2 \mid & \left(B\left(u_{v_{n}}(s), u_{v_{n}}(s)\right)-B\left(u_{v}(s), u_{v}(s)\right), w_{v_{n}}(s)\right) \mid \\
& =2\left|\left(B\left(w_{v_{n}}(s), u_{v}(s)\right), w_{v_{n}}(s)\right)\right| \\
& \leq 2\left\|B\left(w_{v_{n}}(s), u_{v}(s)\right)\right\|_{V^{\prime}}\left\|w_{v_{n}}(s)\right\| \\
& \leq 2\left|w_{v_{n}}(s)\right|\left|u_{v}(s)\right|\left\|w_{v_{n}}(s)\right\| \\
& \leq \frac{\nu}{2}\left\|w_{v_{n}}(s)\right\|^{2}+\frac{2}{\nu}\left|w_{v_{n}}(s)\right|^{2}\left|u_{v}(s)\right|^{2} .
\end{aligned}
$$

Also notice that,

$$
\begin{aligned}
& \left|\int_{0}^{t}\left(\sigma\left(s, u_{v_{n}}(s)\right) v_{n}(s)-\sigma\left(s, u_{v}(s)\right) v(s), w_{v_{n}}(s)\right) \mathrm{d} s\right| \\
& \leq \int_{0}^{t}\left|\left(\left(\sigma\left(s, u_{v_{n}}(s)\right)-\sigma\left(s, u_{v}(s)\right)\right) v_{n}(s), w_{v_{n}}(s)\right)\right| \mathrm{d} s \\
& \quad+\left|\int_{0}^{t}\left(\sigma\left(s, u_{v}(s)\right)\left(v_{n}(s)-v(s)\right), w_{v_{n}}(s)\right) \mathrm{d} s\right| \\
& \leq \quad \sqrt{L} \int_{0}^{t}\left\|w_{v_{n}}(s)\right\|\left|w_{v_{n}}(s)\right|\left|v_{n}(s)\right|_{0} \mathrm{~d} s \\
& \quad+\left|\int_{0}^{t}\left(\sigma\left(s, u_{v}(s)\right)\left(v_{n}(s)-v(s)\right), w_{v_{n}}(s)\right) \mathrm{d} s\right| \\
& \leq \frac{\nu}{4} \int_{0}^{t}\left\|w_{v_{n}}(s)\right\|^{2} \mathrm{~d} s+\frac{L}{\nu} \int_{0}^{t}\left|w_{v_{n}}(s)\right|^{2}\left|v_{n}(s)\right|_{0}^{2} \mathrm{~d} s \\
& \quad+\sup _{0 \leq t \leq T}\left|\int_{0}^{t}\left(\sigma\left(s, u_{v}(s)\right)\left(v_{n}(s)-v(s)\right), w_{v_{n}}(s)\right) \mathrm{d} s\right| .
\end{aligned}
$$

By the boundedness of $\left\{\left|w_{v_{n}}(s)\right|^{2}\right\}$ in $C(0, T ; H)$, and using the Lemma 4.3, the second integral on the right side of (4.16) goes to 0 as $n \rightarrow \infty$. Therefore, given any $\epsilon>0$, there exists an integer $N$ large so that for all $n \geq N$,

$$
\sup _{0 \leq t \leq T}\left|\int_{0}^{t}\left(\sigma\left(s, u_{v}(s)\right)\left(v_{n}(s)-v(s)\right), w_{v_{n}}(s)\right) d s\right|<\epsilon / 2 .
$$

Consider,

$$
C_{L, \nu}=\max \left\{\frac{2}{\nu}, \frac{2 L}{\nu}\right\}
$$


Applying (4.15), (4.16) and (4.17) in (4.14), one obtains for $n \geq N$,

$$
\left|w_{v_{n}}(t)\right|^{2}+\nu \int_{0}^{t}\left\|w_{v_{n}}(s)\right\|^{2} \mathrm{~d} s \leq C_{L, \nu} \int_{0}^{t}\left|w_{v_{n}}(s)\right|^{2}\left(\left|u_{v}(s)\right|^{2}+\left|v_{n}(s)\right|_{0}^{2}\right) \mathrm{d} s+\epsilon
$$

Hence by Gronwall's inequality,

$$
\sup _{0 \leq t \leq T}\left|w_{v_{n}}(t)\right|^{2}+\nu \int_{0}^{T}\left\|w_{v_{n}}(t)\right\|^{2} \mathrm{~d} t \leq \epsilon e^{C_{L, \nu} \int_{0}^{T}\left(\left|u_{v}(t)\right|^{2}+\left|v_{n}(t)\right|_{0}^{2}+1\right) \mathrm{d} t} .
$$

The arbitrariness of $\epsilon$ finishes the proof.

Remark 4.5. From Theorem 4.1 one can see that the equation

$$
\begin{aligned}
& \mathrm{d} u_{v^{\varepsilon}}^{\varepsilon}(t)+\left[A u_{v^{\varepsilon}}^{\varepsilon}(t)+B\left(u_{v^{\varepsilon}}^{\varepsilon}(t), u_{v^{\varepsilon}}^{\varepsilon}(t)\right)\right] \mathrm{d} t \\
& \quad=\left[f(t)+\sigma\left(t, u_{v^{\varepsilon}}^{\varepsilon}(t)\right) v^{\varepsilon}(t)\right] \mathrm{d} t+\sqrt{\varepsilon} \sigma\left(t, u_{v^{\varepsilon}}^{\varepsilon}(t)\right) \mathrm{d} W(t),
\end{aligned}
$$

with $u_{v^{\varepsilon}}^{\varepsilon}(0)=\xi \in H$, has unique strong solution in $\mathrm{L}^{2}(\Omega ; X)$.

As before, the solution admits a representation $u_{v^{\varepsilon}}^{\varepsilon}=\mathcal{G}^{\varepsilon}(W(\cdot)+$ $\left.\frac{1}{\sqrt{\varepsilon}} \int_{0}^{\cdot} v^{\varepsilon}(s) \mathrm{d} s\right)$ by pathwise uniqueness of the solution, and the Girsanov theorem. equation

For all $v \in \mathrm{L}^{2}\left(0, T ; H_{0}\right)$, let $u_{v}$ be the solution of the deterministic control

$$
\mathrm{d} u_{v}(t)+\left[\nu A u_{v}(t)+B\left(u_{v}(t), u_{v}(t)\right)\right] \mathrm{d} t=f(t) \mathrm{d} t+\sigma\left(t, u_{v}(t)\right) v(t) \mathrm{d} t,
$$

with initial condition $u_{v}(0)=\xi \in H$.

Note that $\int_{0}^{\cdot} v(s) d s \in C\left([0, T]: H_{0}\right)$. Define $\mathcal{G}^{0}: C\left([0, T]: H_{0}\right) \rightarrow X$ by

$$
\mathcal{G}^{0}(h)=u_{v} \text { if } h=\int_{0} v(s) \mathrm{d} s \text { for some } v \in L^{2}\left([0, T]: H_{0}\right) .
$$

If $h$ cannot be represented as above, then define $\mathcal{G}^{0}(h)=0$.

Theorem 4.6 (Weak convergence). Let $\left\{v^{\varepsilon}: \varepsilon>0\right\} \subset \mathcal{A}_{M}$ converges in distribution to $v$ with respect to the weak topology on $\mathrm{L}^{2}\left(0, T ; H_{0}\right)$. Then $\mathcal{G}^{\varepsilon}(W(\cdot)+$ $\left.\frac{1}{\sqrt{\varepsilon}} \int_{0}^{\cdot} v^{\varepsilon}(s) \mathrm{d} s\right)$ converges in distribution to $\mathcal{G}^{0}\left(\int_{0}^{\cdot} v(s) \mathrm{d} s\right)$ in $X$, as $\varepsilon \rightarrow 0$.

Proof. Since $S_{M}$ is Polish, the Skorokhod representation theorem can be introduced to construct processes $\left(\tilde{v}^{\varepsilon}, \tilde{v}, \tilde{W}^{\varepsilon}\right)$ such that the distribution of $\left(\tilde{v}^{\varepsilon}, \tilde{v}, \tilde{W}^{\varepsilon}\right)$ is same as that of $\left(v^{\varepsilon}, v, W\right)$, and $\tilde{v}^{\varepsilon} \rightarrow \tilde{v}$ a.s. in the weak topology of $S_{M}$. Thus $\int_{0}^{t} \tilde{v}^{\varepsilon}(s) \mathrm{d} s \rightarrow \int_{0}^{t} \tilde{v}(s) \mathrm{d} s$ weakly in $H_{0}$ a.s. for all $t \in[0, T]$. Without any loss of generality, we will write $\left(v^{\varepsilon}, v, W\right)$ in what follows, though strictly speaking, one should write $\left(\tilde{v}^{\varepsilon}, \tilde{v}, \tilde{W}^{\varepsilon}\right)$.

Let $w_{v^{\varepsilon}}^{\varepsilon}(t)=u_{v^{\varepsilon}}^{\varepsilon}(t)-u_{v}(t)$. We need to prove,

$$
\sup _{0 \leq t \leq T}\left|w_{v^{\varepsilon}}^{\varepsilon}(t)\right|^{2}+\int_{0}^{T}\left\|w_{v^{\varepsilon}}^{\varepsilon}(t)\right\|^{2} \mathrm{~d} t \rightarrow 0
$$

in probability as $\varepsilon \rightarrow 0$. 
Notice that, applying similar estimate as in previous Theorem, equation (4.18), one can get

$$
\begin{aligned}
& \left|w_{v^{\varepsilon}}^{\varepsilon}(t)\right|^{2}+\nu \int_{0}^{t}\left\|w_{v^{\varepsilon}}^{\varepsilon}(s)\right\|^{2} \mathrm{~d} s \\
& \leq 3 C_{L, \nu} \int_{0}^{t}\left|w_{v^{\varepsilon}}^{\varepsilon}(s)\right|^{2}\left(\left|u_{v}(s)\right|^{2}+\left|v^{\varepsilon}(s)\right|_{0}^{2}+1\right) \mathrm{d} s \\
& \quad+\int_{0}^{t}\left|\sigma\left(s, u_{v}(s)\right)\left(v^{\varepsilon}(s)-v(s)\right)\right|^{2} \mathrm{~d} s+\varepsilon K \int_{0}^{t}\left(1+\left\|u_{v^{\varepsilon}}^{\varepsilon}(s)\right\|^{2}\right) \mathrm{d} s \\
& \quad+2 \sqrt{\varepsilon} \int_{0}^{t}\left(\sigma\left(s, u_{v^{\varepsilon}}^{\varepsilon}(s)\right), w_{v^{\varepsilon}}^{\varepsilon}(s)\right) \mathrm{d} W(s) .
\end{aligned}
$$

We take supremum in $0 \leq t \leq T$, then expectation on the above inequality, and use similar estimate (with the help of Burkholder-Davis-Gundy inequality) as in (3.21) on the last term of the right hand side to get,

$$
\begin{aligned}
E\left[\sup _{0 \leq t \leq T}\left|w_{v^{\varepsilon}}^{\varepsilon}(t)\right|^{2}+\nu \int_{0}^{T}\left\|w_{v^{\varepsilon}}^{\varepsilon}(t)\right\|^{2} \mathrm{~d} t\right] \\
\leq 3 C_{L, \nu} E\left[\int_{0}^{T} \sup _{0 \leq t \leq T}\left|w_{v^{\varepsilon}}^{\varepsilon}(t)\right|^{2}\left(\left|u_{v}(t)\right|^{2}+\left|v^{\varepsilon}(t)\right|_{0}^{2}+1\right) \mathrm{d} t\right] \\
\quad+\int_{0}^{T}\left|\sigma\left(t, u_{v}(t)\right)\left(v^{\varepsilon}(t)-v(t)\right)\right|^{2} \mathrm{~d} t+\varepsilon K(C+T) \\
\quad+\sqrt{2 \varepsilon} K\left(C+T+E\left[\sup _{0 \leq t \leq T}\left|w_{v^{\varepsilon}}^{\varepsilon}(t)\right|^{2}\right]\right) .
\end{aligned}
$$

Assume that $\varepsilon<\frac{1}{2 K^{2}}$. Then the Gronwall inequality yields

$$
\begin{aligned}
E & \left.\sup _{0 \leq t \leq T}\left|w_{v^{\varepsilon}}^{\varepsilon}(t)\right|^{2}+\nu \int_{0}^{T}\left\|w_{v^{\varepsilon}}^{\varepsilon}(t)\right\|^{2} \mathrm{~d} t\right] \\
\leq & \left((\varepsilon+\sqrt{2 \varepsilon}) K(C+T)+\int_{0}^{T}\left|\sigma\left(t, u_{v}(t)\right)\left(v^{\varepsilon}(t)-v(t)\right)\right|^{2} \mathrm{~d} t\right) \\
& \times e^{3 C_{L, \nu} \int_{0}^{T}\left(\left|u_{v}(t)\right|^{2}+\left|v^{\varepsilon}(t)\right|_{0}^{2}+1\right) \mathrm{d} t} .
\end{aligned}
$$

Since $v^{\varepsilon} \rightarrow v$ a.s. in the weak topology of $S_{M}$, it is clear from the Eq. (4.24) that as $\varepsilon \rightarrow 0$,

$$
E\left[\sup _{0 \leq t \leq T}\left|w_{v^{\varepsilon}}^{\varepsilon}(t)\right|^{2}+\nu \int_{0}^{T}\left\|w_{v^{\varepsilon}}^{\varepsilon}(t)\right\|^{2} \mathrm{~d} t\right] \rightarrow 0
$$


Let $\delta>0$ be any arbitrary number. Then by Markov's inequality

$$
\begin{aligned}
& P\left\{\sup _{0 \leq t \leq T}\left|w_{v^{\varepsilon}}^{\varepsilon}(t)\right|^{2}+\nu \int_{0}^{T}\left\|w_{v^{\varepsilon}}^{\varepsilon}(t)\right\|^{2} \mathrm{~d} t \geq \delta\right\} \\
& \leq \frac{1}{\delta} E\left[\sup _{0 \leq t \leq T}\left|w_{v^{\varepsilon}}^{\varepsilon}(t)\right|^{2}+\nu \int_{0}^{T}\left\|w_{v^{\varepsilon}}^{\varepsilon}(t)\right\|^{2} \mathrm{~d} t\right] \rightarrow 0 \text { as } \varepsilon \rightarrow 0 .
\end{aligned}
$$

Thus

$$
\sup _{0 \leq t \leq T}\left|u_{v^{\varepsilon}}^{\varepsilon}(t)-u_{v}(t)\right|^{2}+\nu \int_{0}^{T}\left\|u_{v^{\varepsilon}}^{\varepsilon}(t)-u_{v}(t)\right\|^{2} \mathrm{~d} t \rightarrow 0
$$

in probability as $\varepsilon \rightarrow 0$. The proof is now complete.

Remark 4.7. Sabra shell model of turbulence is the other well accepted model in the literature, and the fundamental difference with the GOY model lies in the number of complex conjugation operators used in the nonlinear terms which are responsible for differences in the phase symmetries of the two models, and as a consequence, Sabra shell model exhibits shorter-ranged correlations than the GOY model (see [24]). The equations of motion of the stochastic Sabra shell model have the following form

$$
\begin{aligned}
& \frac{\mathrm{d} u_{n}}{\mathrm{~d} t}+\nu k_{n}^{2} u_{n}+i\left(a k_{n+1} u_{n+2} u_{n+1}^{\star}+b k_{n} u_{n+1} u_{n-1}^{\star}\right. \\
& \left.\quad-c k_{n-1} u_{n-1} u_{n-2}\right)=f_{n}+\sigma_{n}\left(t, u_{n}\right) \frac{\mathrm{d} w_{n}(t)}{\mathrm{d} t}, \quad \text { for } n=1,2, \ldots,
\end{aligned}
$$

along with the boundary conditions

$$
u_{-1}=u_{0}=0 .
$$

Under the same assumptions on the noise and noise coefficient given in Sect. 3, and under the same functional setting, the existence and uniqueness of the strong solution can be established in $\mathrm{L}^{2}\left(\Omega ; C(0, T ; H) \cap \mathrm{L}^{2}(0, T ; V)\right)$. Moreover, by proceeding in the similar fashion as in Sect. 4 , one can easily verify the key estimates and prove the large deviation principle for the solution of the stochastic Sabra model in the Polish space $C(0, T ; H) \cap \mathrm{L}^{2}(0, T ; V)$.

\section{Acknowledgments}

The first author would like to thank Institut Mittag-Leffler (The Royal Swedish Academy of Sciences) for their warm hospitality and support during the visit in September-October 2007, where this work was initiated. He also wants to thank Max-Planck Institute for Mathematics in the Sciences in Leipzig, Germany for providing support and excellent research environment which helped to complete this work. The second author would like to thank the Army Research Office, Probability and Statistics Program for their grant (DODARMY41712). 
Open Access. This article is distributed under the terms of the Creative Commons Attribution Noncommercial License which permits any noncommercial use, distribution, and reproduction in any medium, provided the original author(s) and source are credited.

\section{References}

[1] Amirdjanova, A., Xiong, J.: Large deviation principle for a stochastic Navier-Stokes equation in its vorticity form for a two-dimensional incompressible flow. Discrete Contin. Dyn. Syst. Ser. B 6(4), 651-666 (2006)

[2] Barbato, D., Barsanti, M., Bessaih, H., Flandoli, F.: Some rigorous results on a stochastic Goy model. J. Stat. Phys. 125(3), 677-716 (2006)

[3] Bensoussan, A., Temam, R.: Equations aux dérivées partielles stochastiques non linéaries(1). Isr. J. Math. 11(1), 95-129 (1972)

[4] Budhiraja, A., Dupuis, P.: A variational representation for positive functionals of infinite dimensional Brownian motion. Probab. Math. Stat. 20, 39-61 (2000)

[5] Capinsky, M., Gatarek, D.: Stochastic equations in Hilbert space with application to Navier-Stokes equations in any dimension. J. Funct. Anal. 126, 26-35 (1994)

[6] Chang, M-H.: Large deviation for Navier-Stokes equations with small stochastic perturbation. Appl. Math. Comp. 76, 65-93 (1996)

[7] Chow, P-L.: Large deviation problem for some parabolic Îto equations. Comm. Pure Appl. Math. 45, 97-120 (1992)

[8] Constantin, P., Levant, B., Titi, E.S.: Analytic study of shell models of turbulence. Phys. D 219(2), 120-141 (2006)

[9] Da Prato, G., Zabczyk, J.: Stochastic Equations in Infinite Dimensions. Cambridge University Press, Cambridge (1992)

[10] Dembo, A., Zeitouni, O.: Large Deviations Techniques and Applications. Springer, New York (2000)

[11] Deuschel, J-D., Stroock, D.W.: Large Deviations. Academic Press, San Diego (1989)

[12] Dunford, N., Schwartz, J.: Linear Operators Interscience Publishers. Wiley, New York (1958)

[13] Dupuis, P., Ellis, R.S.: A Weak Convergence Approach to the Theory of Large Deviations. Wiley-Interscience, New York (1997)

[14] Ellis, R.S.: Entropy, Large Deviations and Statistical Mechanics. Springer, New York (1985)

[15] Flandoli, F., Gatarek, D.: Martingale and stationary solutions for stochastic Navier-Stokes equations. Prob. Theory Rel. Fields. 102, 367-391 (1995) 
[16] Fleming, W.H.: A stochastic control approach to some large deviations problems. Recent Mathematical Methods. In: Dolcetta, C., Fleming, W.H., Zolezzi, T. (eds.) Dynamic Programming. Lecture Notes in Math., vol 1119, pp. 52-66. Springer, Heidelberg (1985)

[17] Freidlin, M.I., Wentzell, A.D.: Random Pertubations of Dynamical Systems. Springer, New York (1984)

[18] Frisch, U.: Turbulence. Cambridge University Press, Cambridge (1995)

[19] Kadanoff, L., Lohse, D., Wang, J., Benzi, R.: Scaling and dissipation in the GOY shell model. Phys. Fluids 7(3), 617-629 (1995)

[20] Kallianpur, G., Xiong, J.: Stochastic Differential Equations in Infinite Dimensional Spaces. Institute of Math. Stat. (1996)

[21] Karatzas, I., Shreve, S.: Brownian Motion and Stochastic Calculus, 2nd edn. Springer, New York (1991)

[22] Krylov, N.V., Rozovskii, B.L.: Stochastic evolution equations. J. Sov. Math. 16, 1233-1277 (1981)

[23] Ladyzhenskaya, O.A.: The Mathematical Theory of Viscous Incompressible Flow. Gordon and Breach, New York (1969)

[24] L'vov, V.S., Podivilov, E., Pomyalov, A., Procaccia, I., Vandembroucq, D.: Improved shell model of turbulence. Phys. Rev. E(3) 58(2), 1811-1822 (1998)

[25] Manna, U., Menaldi, J.L., Sritharan, S.S.: Stochastic Analysis of Tidal Dynamics Equation. Infinite Dimensional Stochastic Analysis, pp. 90-113, QP-PQ: Quantum Probab. White Noise Anal., 22, World Science, Hackensack (2008)

[26] Manna, U., Menaldi, J.L., Sritharan, S.S.: Stochastic 2-D Navier-Stokes equation with artificial compressibility. Commun. Stoch. Anal. 1(1), 123-139 (2007)

[27] Menaldi, J.L., Sritharan, S.S.: Stochastic 2-D Navier-Stokes Equation. Appl. Math. Optim. 46, 31-53 (2002)

[28] Metivier, M.: Stochastic Partial Differential Equations in Infinite Dimensional Spaces. Quaderni, Scuola Normale Superiore, Pisa (1988)

[29] Ohkitani, K., Yamada, M.: Temporal intermittency in the energy cascade process and local Lyapunov analysis in fully developed model of turbulence. Prog. Theor. Phys. 89, 329-341 (1989)

[30] Pardoux, E.: Equations aux derivées partielles stochastiques non linéaires monotones. Etude de solutions fortes de type Itô; Thése Doct. Sci. Math. Univ. Paris Sud (1975)

[31] Pardoux, E.: Sur des equations aux dérivées partielles stochastiques monotones. C. R. Acad. Sci. 275(2), A101-A103 (1972) 
[32] Quastel, J., Yau, H.-T.: Lattice gases, large deviations, and the incompressible Navier-Stokes equations. Ann. Math. 2nd Ser.148(1), 51-108 (1998)

[33] Sowers, R.: Large deviations for a reaction diffusion equation with non-Gaussian perturbations. Ann. Probab. 20, 504-537 (1992)

[34] Sritharan, S.S., Sundar, P.: Large deviations for the two-dimensional Navier-Stokes equations with multiplicative noise. Stoc. Process. Appl. 116, 1636-1659 (2006)

[35] Stroock, D.: An Introduction to the Theory of Large Deviations. Springer, Universitext (1984)

[36] Temam, R.: Navier-Stokes Equations, Theory and Numerical Analysis. North-Holland, Amsterdam (1984)

[37] Varadhan, S.R.S.: Large deviations and its Applications, 46, CBMS-NSF Series in Applied Mathematics, SIAM, Philadelphia (1984)

[38] Vishik, M.J., Fursikov, A.V.: Mathematical Problems of Statistical Hydromechanics. Kluwer, Boston (1980)

U. Manna

Max Planck Institute for Mathematics in the Sciences,

Leipzig, Germany.

e-mail: manna@mis.mpg.de

S. S. Sritharan

Graduate School of Engineering and Applied Sciences,

Naval Postgraduate School,

Monterey, USA.

e-mail: sssritha@nps.edu

P. Sundar

Department of Mathematics,

Louisiana State University,

Baton Rouge, USA.

e-mail: sundar@math.lsu.edu

Received: 6 February 2008.

Accepted: 18 March 2009. 